\title{
Superabsorbing metamaterial wormhole: Physical modeling and wave interaction effects
}

\author{
Stanislav I. Maslovski, ${ }^{*}$ Hugo R. L. Ferreira, and Iurii O. Medvedev \\ Instituto de Telecomunicações (Coimbra) \\ DEEC FCTUC Pólo II - Pinhal de Marrocos \\ 3030-290 Coimbra, Portugal \\ Nuno G. B. Brás \\ Instituto de Telecomunicações (Lisboa) \\ Instituto Superior Técnico, Av. Rovisco Pais 1 \\ 1049-001 Lisboa, Portugal, and \\ Universidade Autónoma de Lisboa \\ Rua Santa Marta 56 - Palácio Dos Condes Do Redondo \\ 1169-023 Lisboa, Portugal
}

(Dated: September 5, 2018)

\begin{abstract}
Conjugate-impedance matched superabsorbers are metamaterial bodies whose effective absorption cross section greatly exceeds their physical dimension. Such objects are able to receive radiation when it is not directly incident on their surface. Here, we develop methods of physical modeling of such structures and investigate interactions of the superabsorbers with passing electromagnetic radiation. The particular superabsorbing structure under study is a wormhole comprised of meshes of loaded transmission lines. A theory of electromagnetic wave propagation and absorption in such metamaterial structures is developed. At the frequency of operation, the structure exhibits greatly enhanced absorption as compared to the black body-type absorber of the same size. Peculiar wave absorption effects such as trapping of nearby passing beams of electromagnetic radiation are demonstrated by numerical simulations. Possible modifications of the wormhole structure under the goal of optimizing absorption while minimizing complexity of the involved metamaterials are discussed. Conjugate-impedance matched superabsorbers may find applications as efficient harvesters of electromagnetic radiation, novel antennas, and sensors.
\end{abstract}

\section{INTRODUCTION}

From wave optics, it is known that the scattering and absorption cross sections of resonant particles can be much greater than that of non-resonant bodies with the same dimensions. ${ }^{1}$ For instance, the extinction cross-section in subwavelength particles exhibiting plasmonic or polaritonic resonances can be orders of magnitude greater than the same for a black-body type absorber of a comparable physical size. ${ }^{2-9}$ Effectively, such resonant particles are able to collect the incident wave power from an area much bigger than their physical cross section.

The same physical principle of optimal resonant absorption is used when designing compact receiving antennas. From the theory of wire antennas ${ }^{10-12}$ it is known that a short wire dipole (with length much smaller than half wavelength) is a rather ineffective receiver unless it is loaded with complex impedance $Z_{\text {load }}(\omega)=Z_{\text {dip }}^{*}(\omega)$, where $Z_{\text {dip }}^{*}(\omega)$ is the complex conjugate of the input impedance of the dipole antenna at the frequency $\omega$. Such a conjugateimpedance matched load compensates for the excess reactance of the short dipole antenna, tunes it in resonance with the incident field, and provides for the maximum of the received power. ${ }^{10}$

The ultimate limit for the effective receiving area of a resonant dipole is $(3 / 8 \pi) \lambda^{2}$ (e.g., Ref. 2 ), where $\lambda$ is the radiation wavelength. Note that this limit is determined by the wavelength rather than by the dimensions of the dipole. If a particle supports higher order multipolar resonances (besides the main electric dipolar mode) at the same frequency $\omega$, its absorption cross section can be made larger than that of a resonant dipole. ${ }^{8,9}$ In fact, it can be shown that there is no ultimate upper limit on the effective absorption cross section of a resonant object when more and more multipolar modes of the object pile up at the same resonant wavelength. Analogous results for the gain and directivity of conjugate-impedance matched antennas have been known for a long time. ${ }^{13,14}$

Cylindrical metamaterial superabsorbers utilizing isotropic double-negative metamaterials are known from literature. ${ }^{15}$ However, their performance is limited to the normal incidence of vertically polarized waves. Perfectly conjugate-impedance matched metamaterials that enable optimal absorption of the incident electromagnetic radiation in arbitrary excitation scenarios have been proposed in Ref. 16. With this principle, a spherical object — "metamaterial thermal black hole" - formed by a medium with simultaneously negative permittivity and permeability ${ }^{17}$ can be constructed to posses arbitrarily large absorption cross section, theoretically, independently of the physical radius of the object. ${ }^{18}$ The required condition is that the double-negative (DNG) metamaterials with arbitrarily small loss and arbitrarily large ranges of permittivity and permeability values are attainable. Such metamaterial superabsorbers, 
if realized in practice, could be used, for example, as efficient harvesters of electromagnetic radiation at microwave frequencies or as super-Planckian radiative heat emitters at infrared and optical frequencies. ${ }^{18,19}$

It is also known that the subwavelength superscattering objects ${ }^{3}$ under plane wave incidence and the metamaterial thermal black holes with optically large radius ${ }^{18,20}$ when illuminated by Gaussian beams exhibit very peculiar behaviors of the Poynting vectors in their vicinity, which so far has been only studied theoretically. Unusual wave effects, such as trapping of nearby passing beams of light, have been predicted for such objects. ${ }^{20}$ Although one may introduce an effective optical "Schwarzschild radius" for such superabsorbers, ${ }^{20}$ they do not actually mimic the behavior of light close to a celestial black hole. Note that the beam trapping effects discussed here are very different from the ones reported for the optical black holes with positive index of refraction and the metamaterial black holes, ${ }^{21-24}$ because, in the latter, a beam has to enter the gradient index medium in order to be captured, while, in our case, the DNG metamaterial body captures the beam propagating in free space nearby the body. In other words, the effective absorption cross section of the optical black holes with positive refractive index does not exceed their physical cross section, in contrast to the superabsorbing structures studied in the present article. It is, therefore, of a great interest to identify ways of physical (both numerical and experimental) modeling of such structures.

In this article, we look for ways of such physical modeling by employing topological equivalence (i.e. homeomorphism) between a superabsorbing conjugate impedance matched object in $n$-dimensional space and a corresponding wormhole structure in $n+1$ dimensions (which is discussed in Sec. II), so that, for instance, the wave absorption and scattering effects on a circular superabsorber in two dimensions can be modeled as for surface waves on an equivalent wormhole structure in three dimensions. The respective wormhole structure can be realized at microwave frequencies with readily available techniques.

This article is structured as follows. In Sec. II, a summary of the known results regarding superabsorbers such as the metamaterial thermal black holes is given, including the details of the coordinate transformations that establish the topological equivalence mentioned in the previous paragraph. In Sec. III, possible approaches for physical modeling of such superabsorbing structures utilizing the wormhole topology are discussed, with the most feasible one being selected as based on meshes of loaded transmission lines, which is supported by the analytical theory for the electromagnetic Bloch waves in the periodic meshes of such lines developed in the same section. In Sec. IV the wormhole structure realization, its theoretical analysis, the simulation methods, and the numerical results are presented (with the implementation details of the simulation methods given in the appendix). Finally, in Sec. V the main conclusions of the present study are drawn.

\section{KNOWN SPHERICAL AND CYLINDRICAL METAMATERIAL SUPERABSORBERS}

From our previous studies, ${ }^{18}$ we know that a spherical metamaterial body with radius $a$ and radially dependent isotropic complex permittivity $\varepsilon(r)=\varepsilon^{\prime}(r)-j \varepsilon^{\prime \prime}(r)$ and permeability $\mu(r)=\mu^{\prime}(r)-j \mu^{\prime \prime}(r)$ [at some frequency $\omega$; the time dependence is assumed to be of the form $\exp (+j \omega t)$, where $j=\sqrt{-1}]$ satisfying

$$
\begin{gathered}
\frac{\varepsilon^{\prime}(r)}{\varepsilon_{0}}=\frac{\mu^{\prime}(r)}{\mu_{0}}=-\frac{a^{2}}{r^{2}}, \\
\left|\frac{\varepsilon^{\prime \prime}(r)}{\varepsilon^{\prime}(r)}\right|=\left|\frac{\mu^{\prime \prime}(r)}{\mu^{\prime}(r)}\right|=\tan \delta \rightarrow 0,
\end{gathered}
$$

has the effective absorption cross-section $\sigma_{\text {abs }}(\omega) \rightarrow \infty$ at the frequency $\omega$, independently of the physical radius of the body $a$. In these relations, $\varepsilon_{0}$ and $\mu_{0}$ are the permittivity and the permeability of the surrounding space, e.g. free space. The parameters $\varepsilon_{0}$ and $\mu_{0}$ are assumed real-valued.

For objects made of the materials with finite values of the loss tangent: $\tan \delta>0$, and a limited variation range of the relative material parameters when $r \rightarrow 0:\left|\varepsilon^{\prime}(r) / \varepsilon_{0}\right|<\infty,\left|\mu^{\prime}(r) / \mu_{0}\right|<\infty$, the absorption cross section is finite, but still it can be large as compared to the physical dimensions of the body: $\sigma_{\text {abs }} \gg \pi a^{2}$, even when $a \gg \lambda$, where $\lambda=2 \pi c / \omega$ is the radiation wavelength (with $c=1 / \sqrt{\varepsilon_{0} \mu_{0}}$ being the speed of light in the surrounding space).

This result can be explained by the fact that a body with the parameters (1) and (2) is conjugate-impedance matched with the surrounding space, at every possible spatial harmonic of the incident field. ${ }^{18}$ In order to prove this fact one can use, for instance, the expansion of an arbitrary incident plane wave into spherical harmonics with the origin at the center of the body, as it was done in Ref. 18. It can be shown that all incident spherical harmonics in this expansion are absorbed by such body without reflections, and thus the body is able to receive all (theoretically, infinite) power transported by a plane wave. This result means that the absorption cross section of such a body is also theoretically infinite. 
An analogous result can be obtained as well in the case of a cylindrical body ${ }^{16}$ with radius $a$ and anisotropic radially dependent material parameters

$$
\frac{\overline{\bar{\varepsilon}}^{\prime}}{\varepsilon_{0}}=\frac{\overline{\bar{\mu}}^{\prime}}{\mu_{0}}=\left(\begin{array}{ccc}
-m & 0 & 0 \\
0 & -\frac{1}{m} & 0 \\
0 & 0 & -\frac{1}{m}\left(\frac{a^{2}}{\rho^{2}}\right)^{1+1 / m}
\end{array}\right)
$$

where the components of the material tensors are given in the cylindrical coordinate system $\left(x_{1}, x_{2}, x_{3}\right) \equiv(\rho, \varphi, z)$, with $\rho$ being the radial distance in this system, and $m>0$ being an arbitrary parameter. When $m=1$, the material parameters (3) become uniaxial with respect to the $z$-axis, and thus are isotropic in the $x y$-plane. Because the latter case is simpler to realize in practice, in what follows, we select $m=1$.

The superabsorbing property of such spherical and cylindrical objects can be also explained with a coordinate transformation (transformation optics ${ }^{25}$ ) technique. ${ }^{16,18}$ Namely, under the coordinate transformation $r \mapsto a^{2} / r$ (in the spherical case) or $\rho \mapsto a^{m+1} / \rho^{m}$ (in the cylindrical case), the media with the parameters (1) and (3) transform into a uniform DNG medium with isotropic parameters $\varepsilon^{\prime}=-\varepsilon_{0}$ and $\mu^{\prime}=-\mu_{0}$, i.e., they transform into the left-handed ${ }^{17}$ counterpart of the surrounding space. The same transformation also maps the region $r<a$ into the region $r>a$ (or $\rho<a$ to $\rho>a$ in the cylindrical case), while keeping the tangential components of the electric and magnetic fields intact at the surface $r=a(\rho=a)$.

Therefore, the plane wave incidence onto a spherical or cylindrical object with the parameters (1) or (3) in a $n$ dimensional space (for the spherical case, $n=3$; for the cylindrical case, $n=2$ ) can be equivalently reformulated as a problem defined on a hypersurface in $(n+1)$-dimensional space, in which the two separate $r>a$ regions - the surrounding space and the transformed material object - are joined on the same $n$-dimensional sphere, on which $r=a$. Topologically, a junction of two such regions or subspaces is a $(n+1)$-dimensional wormhole.

When $\tan \delta \rightarrow 0$, the material parameters $\varepsilon(\omega)$ and $\mu(\omega)$ in these two joined regions differ only by sign. Therefore, any time-harmonic solution (with frequency $\omega$ ) of the uniform Maxwell equations in the first region has a time-reversal "mirror" solution in the second region. From here it follows that if the tangential components of $\mathbf{E}$ and $\mathbf{H}$ in both subspaces are continuous at the interface $r=a$ when passing from one subspace to another, any wave propagating from $r=\infty$ towards $r=a$ in the first region will be continued by a wave propagating from $r=a$ towards $r=\infty$ in the second region, without any reflections at the interface. Thus, this observation allows for an alternative explanation of the superabsorption phenomenon and, as will be shown later, also provides us with an ability to demonstrate the superabsorption effect in practice.

\section{TOWARDS PHYSICAL MODELING OF SUPERABSORPTION EFFECT IN 2D}

Unfortunately, with the facilities that are currently at our hands, a practical demonstration of the superabsorption effect in three dimensions appears to be rather difficult. Therefore, here we aim at physical modeling of this effect in a setup with reduced dimensionality, namely, in just two dimensions (2D).

Indeed, the case of the cylindrical object mentioned in Sec. II reduces to an effectively 2D case when the wave vector of an incident wave lies in the $x y$-plane. In this case, an incident wave of the transverse electric (TE) polarization has the electric field vector $\mathbf{E}^{\text {inc }}$ parallel to the $z$-axis, and the magnetic field vector $\mathbf{H}^{\text {inc }}$ in the $x y$-plane. Conversely, an incident wave of the transverse magnetic (TM) polarization has the magnetic field vector along the $z$-axis and the electric field vector in the $x y$-plane. The fields are independent of the $z$-coordinate.

Note that the $z z$-component of the permeability tensor $\overline{\bar{\mu}}$ has no influence on such waves of the TE polarization (respectively, the $z z$-component of the permittivity tensor $\overline{\bar{\varepsilon}}$ has no effect on the waves of the TM polarization). In particular, this means that the superabsorption effect in the $2 \mathrm{D}$ case can be demonstrated for the waves of the TE polarization by, for example, using a double negative (DNG) metamaterial with isotropic negative permeability (which is the case of Ref. 15), or even a metamaterial which is non-magnetic along the $z$-axis.

Moreover, for the waves of the TE polarization, only the $z z$-component of $\overline{\bar{\varepsilon}}$ matters, because in such waves the electric field is oriented along the $z$-axis. Hence, the in-plane $x y$ components of the dielectric tensor can have arbitrary values without affecting the performance of the superabsorber for the incident waves of this polarization.

Based on these considerations, one may think of using the well-known uniaxial metamaterial designs ${ }^{26}$ when realizing the superabsorber. In such designs, the negative dielectric permittivity is realized with metallic rods or strips, and the negative magnetic response is due to split ring resonators (SRR). There is, however, a disadvantage in these designs: because the magnetic response is realized with a resonant inclusion (the SRR), the loss tangent of the effective magnetic permeability of such metamaterials is relatively high. Additionally, the realizable range of $\left|\mu_{\mathrm{eff}}\right|$ in such media is rather low: It is usually hard to obtain $\left|\mu_{\text {eff }} / \mu_{0}\right| \gtrsim 3$ while maintaining a reasonably small $\tan \delta$ (the latter limitation is less critical in the $2 \mathrm{D}$ case, because, in this case, one has to realize a material with $\left.\mu_{\rho}^{\prime} / \mu_{0}=\mu_{\varphi}^{\prime} / \mu_{0}=-1\right)$. 
These considerations have led us to a realization based on considerably different ideas, which is discussed in the next section.

\section{A. Superabsorbing TL-based wormhole structure}

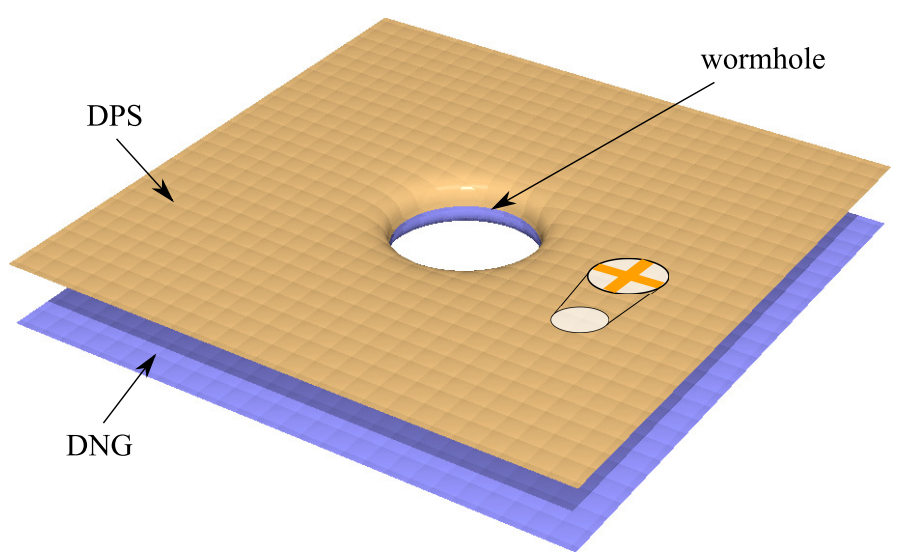

FIG. 1. (Color online) Schematic representation of a TL-based wormhole structure formed by two electrically connected two-dimensional DPS and DNG domains. The DPS and DNG domains are realized by employing TL-based metamaterials, which are 2D meshes of loaded TLs. In this figure the domains are shown as golden (top) and blue (bottom) surfaces of zero thickness, although, in a real structure, the two meshes will always have finite thickness. The two TL meshes are electrically connected at the circumference $\rho=a$ of the wormhole neck.

The limitations of the conventional metamaterials discussed above can be overcome when using $2 \mathrm{D}$ metamaterials realized with meshes of loaded transmission lines (TL). The theory of such 2D metamaterials has been developed in a number of works (for a review, see Ref. 27). There is also a possibility to extend such concepts to 3D. ${ }^{28-30}$ The electromagnetic waves in such structures are represented by waves of electric currents and voltages in the TL segments.

Moreover, as it was mentioned in Sec. II, a conjugate-impedance matched $n$-dimensional superabsorber can be modeled with a wormhole structure in space with $(n+1)$ dimensions. Replacing a highly nonuniform DNG metamaterial object by an equivalent wormhole "tunnel" to a uniform DNG subspace greatly reduces the realization costs and complexity while preserving all observable physical phenomena associated with the original structure.

In our case, the 2D superabsorption effect can be modeled by a wormhole structure composed of two separate TL meshes: the DPS mesh and the DNG mesh, electrically connected at the circumference of the wormhole. This structure is shown in Fig. 1. In what follows, we discuss the realization of such $2 \mathrm{D}$ metamaterials and derive conditions under which the DPS and DNG domains in the top and bottom halves of the structure shown in Fig. 1 are conjugateimpedance matched, which is necessary for the superabsorption effect to occur.

\section{B. Realizing DPS and DNG domains}

The unit cell of a generic 2D TL-based metamaterial is shown in Fig. 2. By selecting proper loads, such a TL-based metamaterial can be made to support forward or backward waves and thus operates effectively as a double positive (DPS) medium with $\varepsilon_{\text {eff }}^{\prime}>0$ and $\mu_{\text {eff }}^{\prime}>0$, or as a DNG medium with $\varepsilon_{\text {eff }}^{\prime}<0$ and $\mu_{\text {eff }}^{\prime}<0$.

The dispersion relation for the 2D plane waves (also called Bloch waves) in a periodic structure with the unit cell shown in Fig. 2 can be obtained with the use of the ABCD matrices of the TL segments, in a manner analogous to what was done in Ref. 29. The result is

$$
\cos \left(k_{x} d\right)+\cos \left(k_{y} d\right)=\frac{Y Z}{4}+\cos \left(\beta_{0} d\right)\left(2+\frac{Y Z}{4}\right)+j \sin \left(\beta_{0} d\right)\left(\frac{Z}{Z_{0}}+\frac{Y Z_{0}}{2}\right) .
$$

Here, $d$ is the size of the (square) unit cell, $\mathbf{k}=\left(k_{x}, k_{y}\right)$ is the wave vector of the propagating Bloch wave, $\beta_{0}$ and $Z_{0}$ are the propagation factor and the characteristic impedance in the (unloaded) TL segments, respectively. These parameters, in general, depend on the frequency: $\beta_{0} \equiv \beta_{0}(\omega), Z_{0} \equiv Z_{0}(\omega)$. 


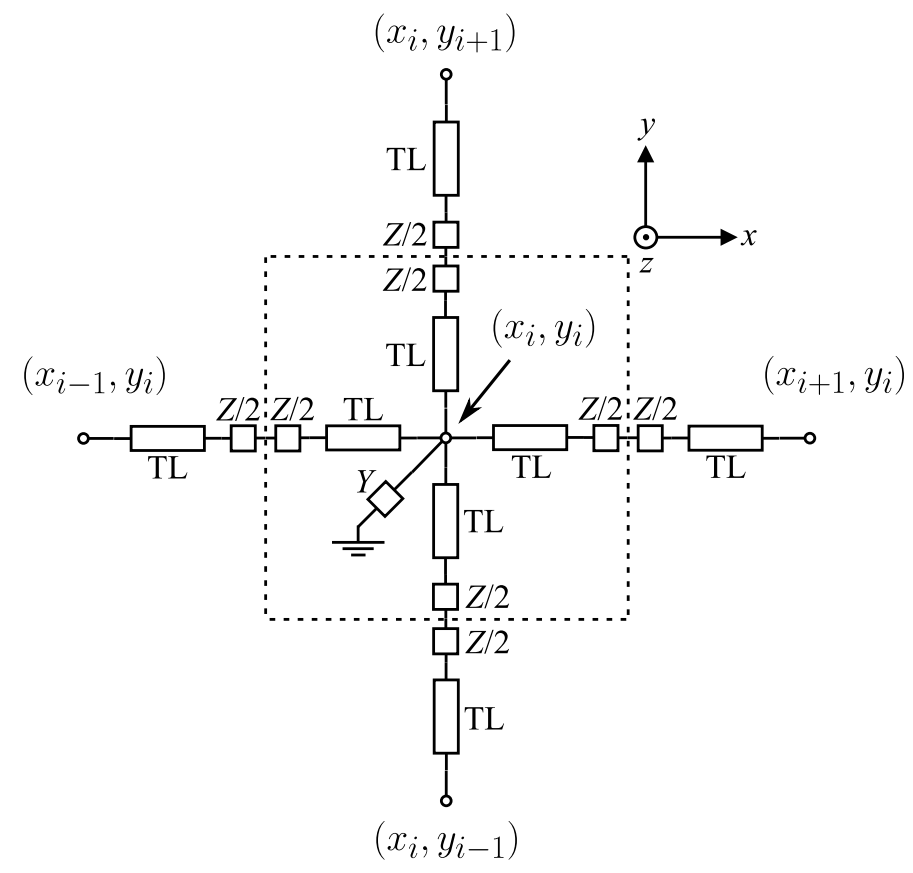

FIG. 2. Circuit diagram of the loaded 2D transmission line (TL) mesh. The unit cell of the structure is indicated with a dashed square. The distance between the centers of the neighboring unit cells is $x_{i+1}-x_{i}=y_{i+1}-y_{i}=d$. Because the load impedance $Z$ at the unit cell edges is shared between the two neighboring cells, it is split in two halves in the schematic: $Z=Z / 2+Z / 2$. The shunt admittance $Y$ connects every node of the TL mesh with the common ground shared by all TL segments. The elements labeled by TL are transmission line segments (e.g., microstrip lines) with the characteristic impedance $Z_{0}$, the propagation factor $\beta_{0}$, and the length $d / 2$.

Considering, for instance, all Bloch waves with a fixed value of the transverse wavenumber $k_{y}$, we can express the longitudinal propagation factor $k_{x}$ as

$$
k_{x}= \pm \frac{1}{d} \cos ^{-1}\left[\frac{Y Z}{4}-\cos \left(k_{y} d\right)+\cos \left(\beta_{0} d\right)\left(2+\frac{Y Z}{4}\right)+j \sin \left(\beta_{0} d\right)\left(\frac{Z}{Z_{0}}+\frac{Y Z_{0}}{2}\right)\right] .
$$

Here the ambiguity in sign of $k_{x}$ is related to the fact that in a reciprocal structure there always exist two waves with the positive and the negative phase velocities which satisfy the same dispersion equation.

We can introduce an analog of the plane wave impedance for such Bloch waves, the Bloch impedance $Z_{\mathrm{B}}$, as a ratio between the line voltage and the $x$-directed current in the TL mesh at any fixed cell boundary $x=$ const (for more details, see Ref. 29). The result is

$$
Z_{\mathrm{B}}= \pm\left(Z_{0} \tan \frac{\beta_{0} d}{2}-\frac{j Z}{2}\right) \cot \frac{k_{x} d}{2}
$$

where $k_{x}$ is given by (5) and the sign must be chosen so that $\operatorname{Re} Z_{\mathrm{B}}>0$.

Let us consider the case when the loads are such that $Z=1 /(j \omega C)$ (i.e., a serial capacitor is inserted between the line ends in the neighboring cells) and $Y=1 /(j \omega L)$ (i.e., there is a shunt inductor to the ground at every line crossing). We can also express the TL parameters $\beta_{0}$ and $Z_{0}$ through the inductance $L_{0}$ and the capacitance $C_{0}$ per unit length of the TL as $\beta_{0}=\omega \sqrt{L_{0} C_{0}}$ and $Z_{0}=\sqrt{L_{0} / C_{0}}$.

Then, in the long wavelength limit, when $|\mathbf{k}| d \ll 1$ and $\beta_{0} d \ll 1$, the solution of the dispersion equation (4) can be approximated as

$$
k_{x}= \pm \sqrt{\omega^{2} L_{\mathrm{eff}} C_{\mathrm{eff}}-k_{y}^{2}}
$$

and the Bloch impedance as

$$
Z_{\mathrm{B}}= \pm \frac{\omega L_{\mathrm{eff}}}{k_{x}}
$$


where $L_{\mathrm{eff}}=L_{0}-1 /\left(\omega^{2} C d\right)$ and $C_{\mathrm{eff}}=2 C_{0}-1 /\left(\omega^{2} L d\right)$.

The situation without loads (i.e. a square mesh of the unloaded TLs) can be modeled with the same equations when $Z=0, Y=0$. In this case, the dispersion equation (4) reduces to

$$
\cos \left(k_{x} d\right)+\cos \left(k_{y} d\right)=2 \cos \left(\beta_{0} d\right),
$$

and the expression for the Bloch impedance (6) to $Z_{\mathrm{B}}= \pm Z_{0} \tan \left(\beta_{0} d / 2\right) \cot \left(k_{x} d / 2\right)$. From here, in the limit when $|\mathbf{k}| d \ll 1$ and $\beta_{0} d \ll 1$, we obtain

$$
k_{x}= \pm \sqrt{2 \omega^{2} L_{0} C_{0}-k_{y}^{2}}
$$

and $Z_{\mathrm{B}}= \pm \omega L_{0} / k_{x}$.

By comparing Eqs. (7) and (8) with analogous expressions for the propagation factor and the wave impedance of the TE-polarized plane waves in continuous media, we can identify the parameters $C_{\text {eff }}$ and $L_{\text {eff }}$ as the analogs of the permittivity $\varepsilon_{\text {eff }}$ and the permeability $\mu_{\text {eff }}$ of such media. Respectively, in the $2 \mathrm{D}$ configuration we are discussing, a TL mesh with no loading and with $C_{\text {eff }}=2 C_{0}, L_{\text {eff }}=L_{0}$, will model the surrounding space with the parameters $\varepsilon_{0}, \mu_{0}$ (the DPS domain), and a loaded TL mesh such that $L_{\text {eff }}<0, C_{\text {eff }}<0$ will model the medium with negative material parameters (the DNG domain).

\section{Conjugate-impedance matching between DPS and DNG domains}

For the superabsorption effect to appear the absorber must be conjugate-impedance matched to the surrounding space. In order to study the possibility to realize this condition in 2D with the TL-based metamaterials, let us consider an interface between a DPS halfplane and a DNG halfplane. Without any loss of generality, we may assume that this interface is located at $x=0$, so that the DPS region is located at $x<0$ and the DNG region is at $x>0$.

In the DPS region, both group and phase velocities of a wave propagating towards the interface (the incident wave) are positive, and thus $\operatorname{Re} k_{x}^{\mathrm{DPS}}>0$ and one has to select the positive branch of Eq. (5). In the DNG region a wave propagating away from the interface (the transmitted wave) has positive group velocity and negative phase velocity. Therefore, $\operatorname{Re} k_{x}^{\mathrm{DNG}}<0$, and one has to select the negative branch of Eq. (5).

The Bloch impedance for the incident wave in the DPS region, therefore, reads

$$
Z_{\mathrm{B}}^{\mathrm{DPS}}=Z_{0}^{\mathrm{DPS}} \tan \frac{\beta_{0}^{\mathrm{DPS}} d}{2} \cot \frac{k_{x}^{\mathrm{DPS}} d}{2},
$$

where we select the plus branch of Eq. (6) in order to have Re $Z_{\mathrm{B}}^{\mathrm{DPS}}>0$. Respectively, the Bloch impedance for the transmitted wave in the DNG region reads

$$
Z_{\mathrm{B}}^{\mathrm{DNG}}=\left(Z_{0}^{\mathrm{DNG}} \tan \frac{\beta_{0}^{\mathrm{DNG}} d}{2}-\frac{j Z}{2}\right) \cot \frac{k_{x}^{\mathrm{DNG}} d}{2},
$$

where the plus sign in front of Eq. (6) is selected because when $L_{\text {eff }}<0$ (which holds in the DNG domain) the real part of the parenthesized expression in Eq. (12) is negative and also $\operatorname{Re} k_{x}^{\mathrm{DNG}}<0$.

The conjugate-impedance match of the DPS and the DNG regions occurs when

$$
Z_{\mathrm{B}}^{\mathrm{DNG}}\left(\omega, k_{y}\right)=\left(Z_{\mathrm{B}}^{\mathrm{DPS}}\left(\omega, k_{y}\right)\right)^{*} .
$$

This equality must hold at a given frequency $\omega$ and at arbitrary real $k_{y}$ in order for the two regions to be matched for all propagating and evanescent spatial harmonics of the incident field with the selected frequency $\omega$.

As follows from Eqs. (11) and (12), the required matching for arbitrary $k_{y}$ can be achieved only when

$$
k_{x}^{\mathrm{DNG}}\left(\omega, k_{y}\right)=-\left(k_{x}^{\mathrm{DPS}}\left(\omega, k_{y}\right)\right)^{*} .
$$

In this case, Eq. (13) can be reduced to

$$
Z=-2 j\left(\left(Z_{0}^{\mathrm{DPS}}\right)^{*} \tan \frac{\left(\beta_{0}^{\mathrm{DPS}}\right)^{*} d}{2}+Z_{0}^{\mathrm{DNG}} \tan \frac{\beta_{0}^{\mathrm{DNG}} d}{2}\right) .
$$


By substituting this expression into Eq. (4) and using Eqs. (9) and (14) we find that in order to achieve matching at all $k_{x}$ the load admittance $Y$ has to be

$$
Y=-\frac{2 j}{\cos ^{2}\left(\beta_{0}^{\mathrm{DNG}} d / 2\right)}\left[\left(\frac{\sin \left(\beta_{0}^{\mathrm{DPS}} d\right)}{Z_{0}^{\mathrm{DPS}}}\right)^{*}+\frac{\sin \left(\beta_{0}^{\mathrm{DNG}} d\right)}{Z_{0}^{\mathrm{DNG}}}\right]
$$

In the case when $\beta_{0}^{\mathrm{DPS}}=\beta_{0}^{\mathrm{DNG}}=\beta_{0}, \operatorname{Im} \beta_{0} \rightarrow 0$, and $Z_{0}^{\mathrm{DPS}}=Z_{0}^{\mathrm{DNG}}=Z_{0}, \operatorname{Im} Z_{0} \rightarrow 0$, Eqs. (15) and (16) reduce to

$$
\begin{aligned}
& Z=-4 j Z_{0} \tan \frac{\beta_{0} d}{2} \\
& Y=-\frac{8 j}{Z_{0}} \tan \frac{\beta_{0} d}{2}
\end{aligned}
$$

When, additionally, $\beta_{0} d \ll 1$, we may approximate these relations as

$$
\begin{aligned}
& Z=-2 j Z_{0} \beta_{0} d=-2 j \omega L_{0} d, \\
& Y=-\frac{4 j}{Z_{0}} \beta_{0} d=-4 j \omega C_{0} d .
\end{aligned}
$$

From here one can see that the conjugate-impedance matching between a dense unloaded DPS TL mesh and a dense loaded DNG TL mesh with negligible loss is achieved when $L_{\mathrm{eff}}^{\mathrm{DNG}}=-L_{\mathrm{eff}}^{\mathrm{DPS}}=-L_{0}$ and $C_{\mathrm{eff}}^{\mathrm{DNG}}=-C_{\mathrm{eff}}^{\mathrm{DPS}}=-2 C_{0}$, which is analogous to the conjugate matching condition for the DPS and DNG regions of continuous media. ${ }^{16}$

\section{WORMHOLE STRUCTURE NUMERICAL MODELING AND ANALYSIS}

In a practical realization of the wormhole structure schematically shown in Fig. 1, one can employ meshes of strip lines with the unit cells depicted in Fig. 3 (a,b). These unit cells fill the DPS and the DNG domains shown in Fig. 1 by the golden (top) and the blue (bottom) surfaces, respectively. In practice, these DPS and DNG networks can be laid atop one another so that they are separated by the common ground plane (for example, the bottom metalization of the DPS network can also serve as the top metalization for the DNG network, or vice versa). Note that in the middle of this structure, where the wormhole is located, there are no strip lines or loads so that no in-plane propagation may happen in that region. Instead, at the perimeter of the wormhole neck, the open ports of the DPS unit cells are electrically connected to the corresponding ports of the DNG unit cells with short vertical metallic strips that pass through the opening in the middle ground layer. Therefore, a wave propagating in the DPS network towards the wormhole neck, after passing through the connections at the circumference of the wormhole neck, will continue to propagate in the DNG region in an outward direction.

In order to numerically analyze such structure composed of many DPS and DNG unit cells, one can either use a general purpose electromagnetic simulator (such as CST Microwave Studio ${ }^{31}$ or ANSYS HFSS ${ }^{32}$ ) or develop a custom software. However, note that the detailed full-wave simulation of a structure with $10^{4}-10^{5}$ unit cells requires a lot of computing resources.

In this work we employ a strategy in which a general purpose simulator is only used to model isolated DPS and DNG unit cells. From these simulations, the scattering parameters (the $S$-parameters) of the unit cells are found. A very good approximation for the same parameters for the main propagating mode can be also obtained analytically by using the TL-based unit cell model from Sec. III B, which is done in Appendix A.

When the $S$-parameters of the cells are known, the behavior of the whole structure formed by many thousands of cells is modeled with an in-house simulator based on the frequency-domain transmission line matrix (FDTLM) method. ${ }^{33}$ In this method, the unit cells are represented as multiport waveguide joints or blocks with a given number of ports (which is four for a structure with square unit cells) and a given number of incident and reflected waves in each port (which is two when only the main modes of a single polarization are considered). The implementation details of the FDTLM method are given in Appendix B. 


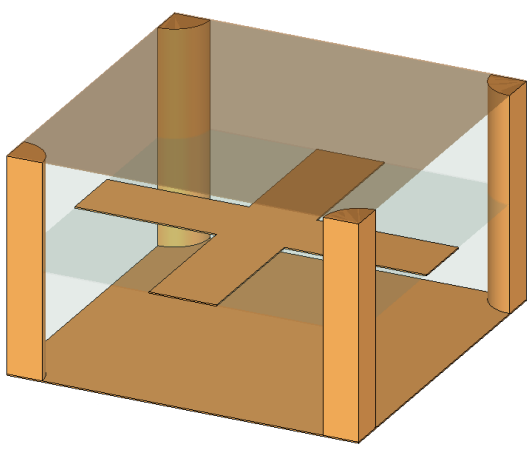

(a)

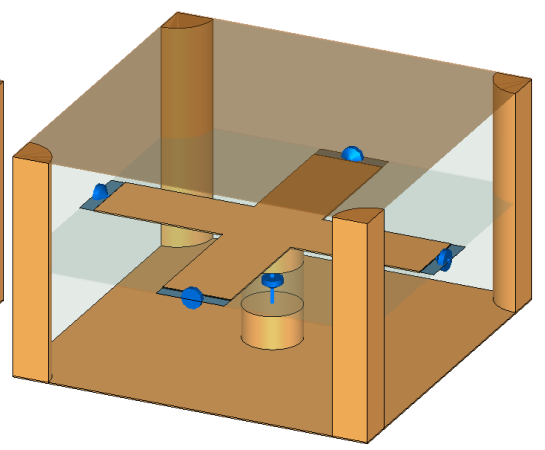

(b)

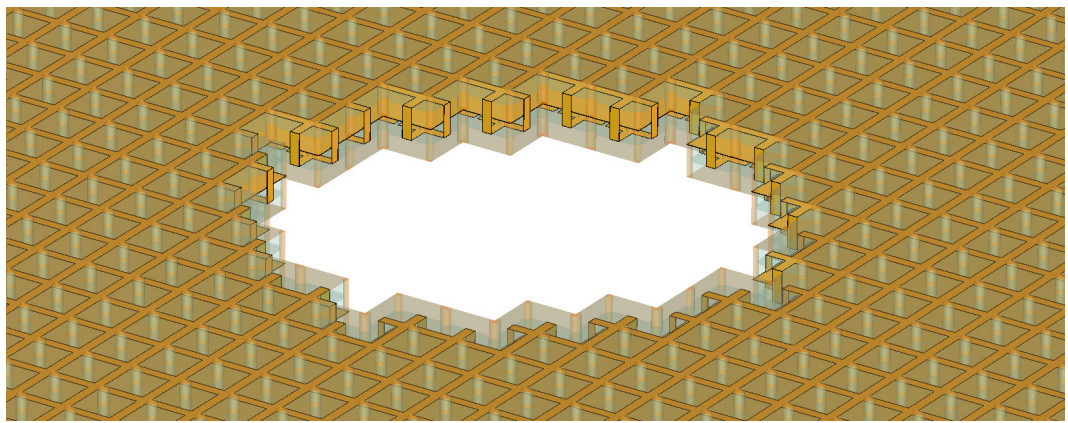

(c)

FIG. 3. (Color online) The unit cells of the DPS [panel (a)] and the DNG [panel (b)] TL meshes. The DPS unit cell is formed by an unloaded crossing of two symmetric strip lines. The DNG unit cell is formed by two crossed stripline segments loaded at the crossing by a lumped inductor connected to the ground (which realizes $Y$ ), and by four lumped capacitors at the edges of the unit cell (which realize $Z / 2$ ). The lumped elements are shown in blue. The ground metalizations at the top (shown semi-transparent) and the bottom are electrically connected by cylindrical metallic pillars at the corners of the unit cell in order to prevent excitation of the unwanted parallel plate waveguide mode. Panel (c) shows a possible realization of the wormhole structure shown in Fig. 1 with these DPS and DNG cells. The top layer is formed by the DPS cells. The bottom layer is formed by the DNG cells. The strip lines in the DPS and DNG cells are electrically connected at the wormhole edge. The pictures are produced with the CST Microwave Studio software.

\section{A. Numerical results of analytical model and CST simulations}

The typical dispersion curves for the Bloch waves propagating in the uniform conjugate-impedance matched DPS and DNG domains realized as meshes of loaded strip lines are shown in Fig. 4. These results are obtained with the analytical model of Sec. III B (AM) and with unit cell simulations in the CST Microwave Studio (CST). In Fig. 4, the normalized free space wavenumber $k_{0} d=(\omega d / c)$ is displayed on the vertical axis, and the normalized Bloch wave propagation factor $q_{x}=k_{x} d$ is displayed on the horizontal axis.

Let us first discuss the results of the AM. For this case, the curves for both real and imaginary parts of $q_{x}$ are shown in Fig. 4. In the considered range of frequencies, the wave dispersion in the DPS network is similar to the dispersion of a plane wave in a dielectric. Indeed, in this example, the relative permittivity of the dielectric that fills the strip lines is $\varepsilon_{r}=3$, and, therefore, as follows from Eq. (10), at low frequencies, $k_{x}=\sqrt{2 \varepsilon_{\mathrm{r}}} k_{0} \approx 2.45 k_{0}$. The wave dispersion in the DNG network exhibits a band gap region at the normalized frequencies $k_{0} d \lesssim 0.075$, where the normalized Bloch wave propagation factor $q_{x}$ is such that $\operatorname{Re} q_{x}= \pm \pi, \operatorname{Im} q_{x} \neq 0$. The region $0.075 \lesssim k_{0} d \lesssim 0.256$ is the backward wave propagation region, in which $q_{x}\left(\partial q_{x} / \partial \omega\right)<0$. The dispersion curves for the waves in the DPS and the DNG networks intersect in this range at $k_{0} d \approx 0.182, q_{x} \approx \pm 0.447$.

At $k_{0} d \approx 0.256$ the backward wave dispersion branch of the DNG network transition into the forward wave dispersion branch of the same network. Normally, a second band gap opens around this point. However, this band gap closes when the characteristic impedances of the DPS and the DNG TL segments match and the values of $L$ and $C$ loads are obtained from the conjugate-impedance match conditions [Eqs. (17) and (18)], which is our case.

The dependence of the Bloch wave impedance of the DPS and DNG domains on the frequency (obtained with the $\mathrm{AM}$ ) is shown in Fig. 5 (a). In the considered range of frequencies, the wave impedance of the DPS network is close to $50 \mathrm{Ohm}$. The wave impedance of the DNG network is purely imaginary in the bandgap region $k_{0} d \lesssim 0.075$. Above this region, $Z_{\mathrm{B}}^{\mathrm{DNG}}$ is real and positive. The curves for $Z_{\mathrm{B}}^{\mathrm{DPS}}$ and $Z_{\mathrm{B}}^{\mathrm{DNG}}$ intersect at the point $k_{0} d \approx 0.182$, which is the 


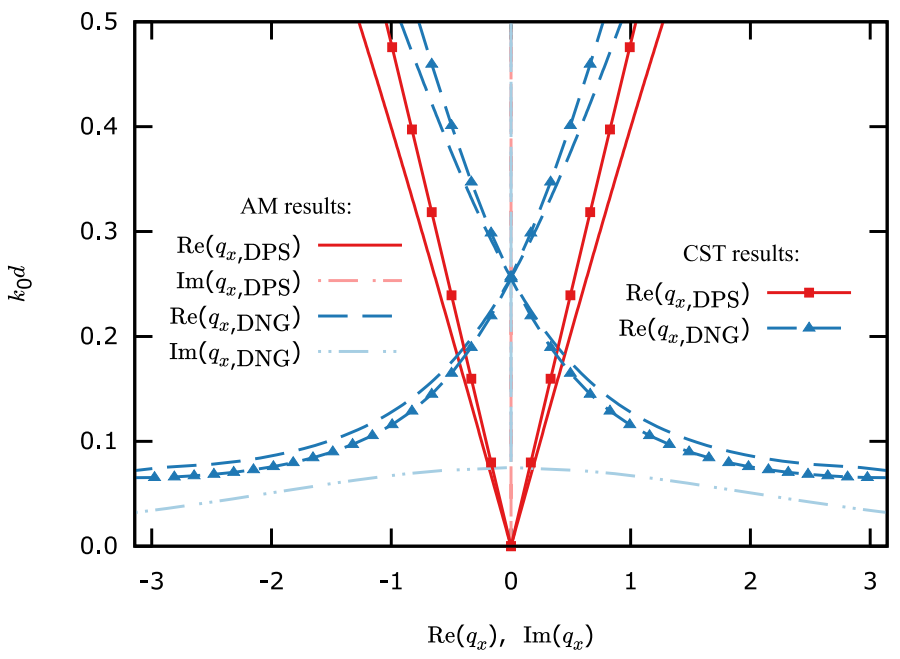

FIG. 4. (Color online) Dispersion of the Bloch waves in the uniform DNG and DPS networks realized by meshes of loaded strip lines obtained with the analytical model (AM) and with the eigenmode solver of the CST Microwave Studio (CST). The propagation direction is along the $x$-axis. Here, $k_{0} d$ is the normalized frequency: $k_{0} d=\omega d / c$ and $q_{x}$ is the normalized propagation factor: $q_{x}=k_{x} d$. The unit cell size is $d=5 \mathrm{~mm}$. The characteristic impedance of the strip lines in both DNG and DPS networks is $Z_{0}=71.6 \mathrm{Ohm}$. The relative permittivity of the dielectric is $\varepsilon_{\mathrm{r}}=3$. In the AM, the load inductance and capacitance in the DNG network are $L=5.16 \mathrm{nH}$, and $C=2.01 \mathrm{pF}$, as found from Eqs. (17) and (18), assuming that, at the operation frequency, $\beta_{0} d=\sqrt{\varepsilon_{\mathrm{r}}} k_{0} d=0.315$. In the CST simulations with the unit cells from Fig. $3(\mathrm{a}, \mathrm{b}), L=5.77 \mathrm{nH}$ and $C=2.11 \mathrm{pF}$.

same as the intersection point of the dispersion curves of the same networks. At this point $Z_{\mathrm{B}}^{\mathrm{DPS}}=Z_{\mathrm{B}}^{\mathrm{DNG}}=50 \mathrm{Ohm}$. Moreover, a direct numerical calculation shows that at this frequency point, the equality $Z_{\mathrm{B}}^{\mathrm{DNG}}=\left(Z_{\mathrm{B}}^{\mathrm{DPS}}\right)^{*}$ holds for the Bloch waves with arbitrary transverse wavenumbers $-\pi / d \leq k_{y} \leq \pi / d$, which confirms that the two domains are perfectly conjugate-impedance matched. The dependency of $Z_{\mathrm{B}}^{\mathrm{DPS}}$ and $Z_{\mathrm{B}}^{\mathrm{DNG}}$ on the transverse wavenumber is depicted in Fig. 5 (b).

The dispersion of the same DPS and DNG cells has also been studied with the eigenmode solver of the CST Microwave Studio. The geometry of the DPS unit cell as realized in the CST Microwave Studio is shown in Fig. 3 (a). Several DNG unit cell models were tested, in which the load capacitance $C$ and the load inductance $L$ were realized by different means. For instance, the capacitance $C$ was realized with a gap in the strip line capped with a metallic patch (to increase the gap capacitance), and the inductance $L$ was realized with a thin helical wire. Realizations using standard surface mounted (SMD) chip components were also checked. It has been found that independently of the way how these loading elements are realized, the modal dispersion resulting from the full wave simulations can be made nearly coincident with the results of the AM (within the frequency range $k_{0} d \lesssim 0.5$ ) by tuning the geometrical parameters of the capacitors (the gap width, the interlacing area) and the inductors (the wire radius, the number of turns). Therefore, in what follows, we discuss the results for the unit cell structure shown in Fig. 3 (b), which models the load inductors and capacitors as effective impedance boundary conditions on an edge or a line within the computational domain of the CST Microwave Studio, as the most general representation of such loads.

The dispersion curves obtained with the CST Microwave Studio eigenmode solver for the DNG and DPS cells with the geometries shown in Fig. 3 (a,b) are depicted in Fig. 4 alongside the AM results. Because the eigenmode solver allows only for calculation of the dispersion of the propagating modes (i.e., the evanescent modes are excluded) this figure only displays the real part of the normalized propagation factor $q_{x}$ as a function of the normalized free space wavenumber $k_{0} d$ for this case. Note that the values of the lumped loads ( $L$ and $C$ ) differ slightly in the CST calculations, as compared to the AM calculations. This is because we have tuned these parameters in order to match the position of the closed band gap in both cases. The observed residual discrepancy is due to simplifications in the AM.

The CST eigenmode solver simulations were performed by considering up to four propagating modes. Fig. 4 shows the dispersion curves for the first mode of the DPS unit cell, and for the first and the third mode of the DNG unit cell. It has to be noted that, for the DNG cell, the CST Microwave Studio eigenmode solver predicts existence of a spurious mode in a very narrow band close to $k_{0} d \approx 0.256$, which is right within the closed bandgap between the backward wave and the forward wave dispersion branches. This spurious mode (the second mode as found by the eigenmode solver) is related to a slight asymmetry in the transmission between the ports $\left(x_{i-1}, y_{i}\right)$ and $\left(x_{i+1}, y_{i}\right)$ [or $\left(x_{i}, y_{i-1}\right)$ and $\left.\left(x_{i}, y_{i+1}\right)\right]$ and the ports $\left(x_{i \pm 1}, y_{i}\right)$ and $\left(x_{i}, y_{i \pm 1}\right)$ (see Fig. 2) in the real unit cell structure depicted in 

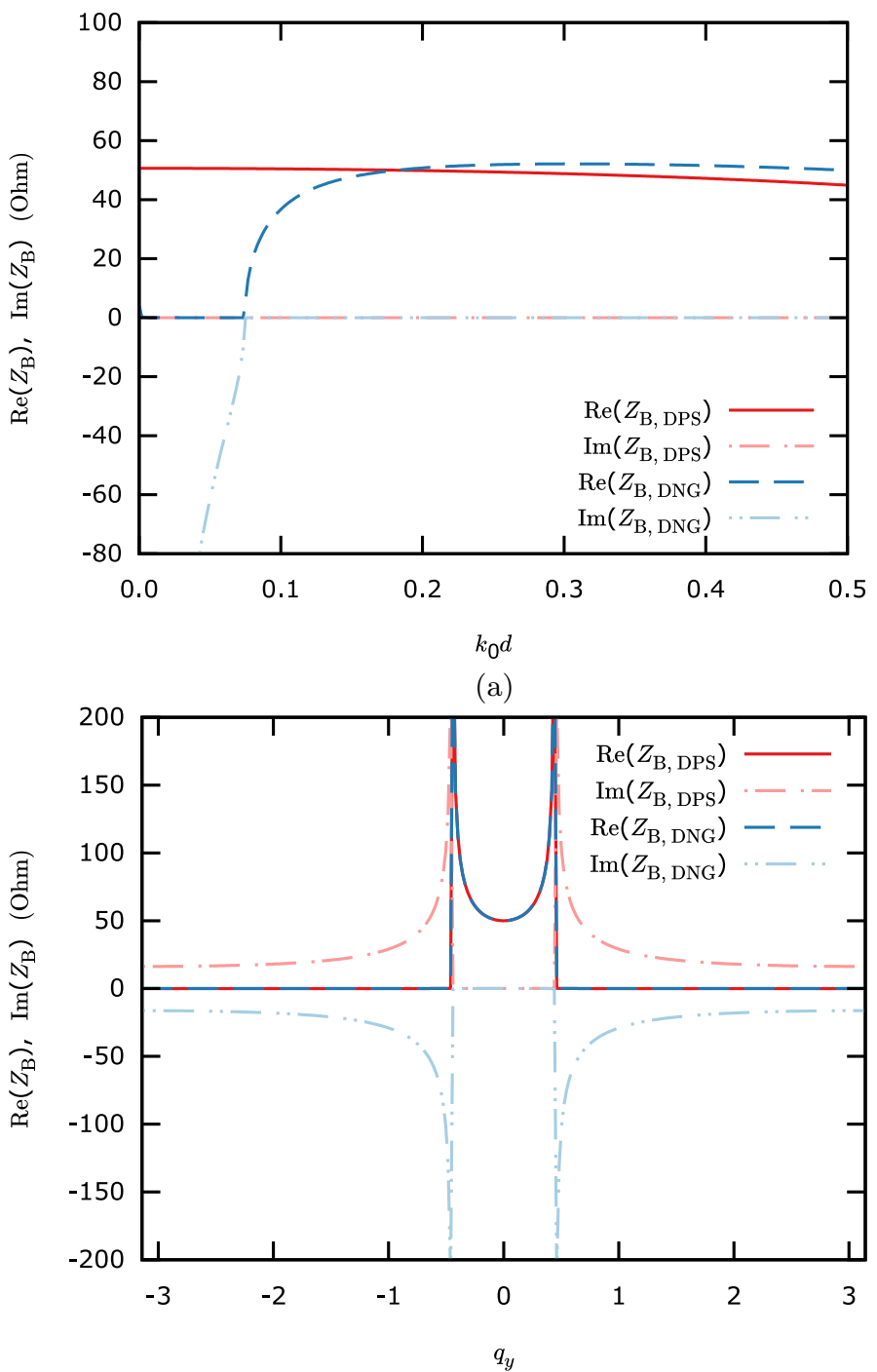

(b)

FIG. 5. (Color online) Panel (a): Bloch wave impedances $Z_{\mathrm{B}}^{\mathrm{DPS}}$ and $Z_{\mathrm{B}}^{\mathrm{DNG}}$ for the waves propagating along the $x$-axis in both DPS and DNG networks as functions of the normalized frequency $k_{0} d$. Panel (b): The same impedances for the conjugate-impedance matched networks as functions of the normalized transverse wavenumber $q_{y}=k_{y} d$, at the normalized frequency $k_{0} d=0.182$. The curves for $\operatorname{Re} Z_{\mathrm{B}}^{\mathrm{DPS}}$ and $\operatorname{Re} Z_{\mathrm{B}}^{\mathrm{DNG}}$ are indistinguishable from each other on the scale of this plot. The parameters used in both panels are the same as in Fig. 4 for the AM.

Fig. 3 (b). This mode is not shown in Fig. 4.

\section{B. Numerical results of FDTLM simulations}

The results of numerical simulations obtained with the FDTLM approach are shown in Fig. 6. In this case, the wormhole structure is formed by the DPS and DNG domains occupying an area of $120 \times 120$ cells (with each cell being a square of size $d \times d$ ) and a wormhole with the radius $R_{\mathrm{WH}}=30 d$. The structure is excited by a plane wave Huygens source (see Appendix B for details) enclosing the whole DPS plane. Hence, Fig. 6 depicts the total (i.e. incident plus scattered) field. The source amplitude is such that $\sqrt{1-\left|\Gamma_{0}\right|^{2}}\left|V_{0}^{\text {inc }}\right|=1$ arb. u., where $V_{0}^{\text {inc }}$ is the incident wave voltage (measured in arb. u.) at the input ports of the unit cells located at $x=-60 d$ and $\Gamma_{0}$ is the Bloch wave reflection coefficient in these ports (see Appendix B).

There are no cells at the middle of the domains where $r<R_{\mathrm{WH}}$. Respectively, in this middle region (shown in white in the figure) there is no propagation. Instead, an incident wave in the DPS domain, when reaching the wormhole, 


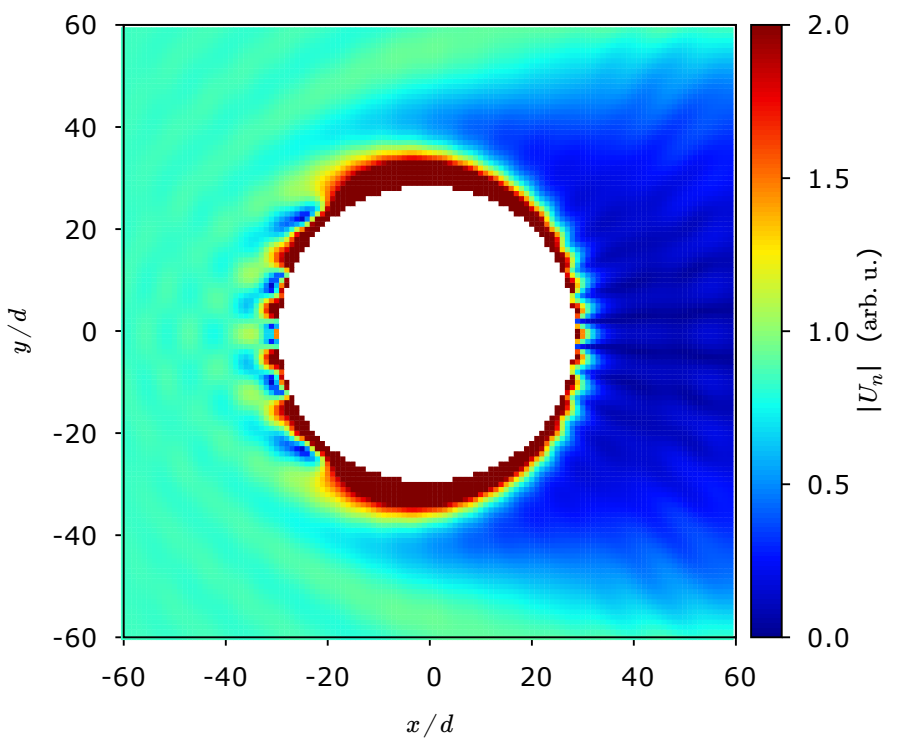

(a)

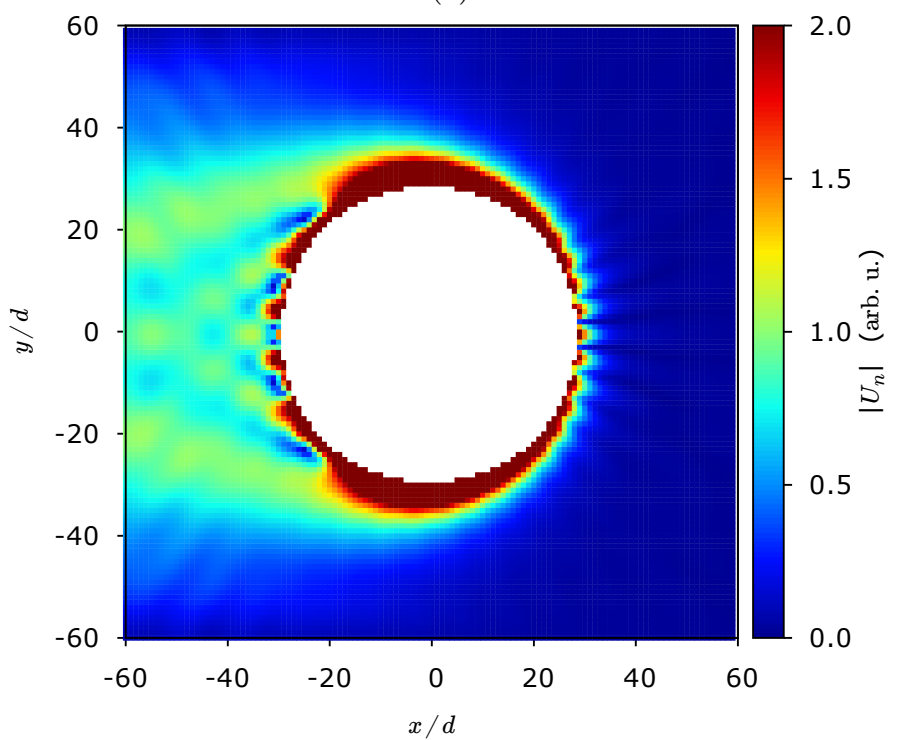

(b)

FIG. 6. (Color online) Distributions of the nodal voltage $\left|U_{n}\right|, n=\lfloor 120(60+y / d)+(60+x / d)\rfloor+1,-60 \leq x / d<60$, $-60 \leq y / d<60$, for the wormhole structure under the plane wave incidence of unitary amplitude (see main text), in both the DPS (a) and the DNG (b) domains as functions of the normalized coordinates $x / d$ and $y / d$. The radius of the wormhole is $R_{\mathrm{WH}}=30 d$. The electrical parameters are: $\beta_{0} d=0.315, \tan \delta=10^{-4}$.

passes to the DNG domain through the connections at the wormhole edge. As is seen from Fig. 6, after passing through the wormhole, the transmitted wave forms a beam that propagates in the DNG domain in the opposite direction with respect to the propagation direction of the incident wave.

One can also see that a shadow is formed in the DPS region behind the wormhole. Moreover, the diameter of the shadow is greater than the diameter of the wormhole, which indicates that the effective absorption cross section of the wormhole is such that

$$
\sigma_{\mathrm{norm}}=\frac{\sigma_{\mathrm{abs}}}{2 R_{\mathrm{WH}}}>1
$$

where $\sigma_{\text {norm }}$ is the normalized absorption cross section and $\sigma_{\text {abs }}$ is given by Eq. (B12) from Appendix B. In this case, the numerically calculated value of $\sigma_{\text {norm }}$ is 1.46, i.e., this metamaterial wormhole object performs about $50 \%$ better than the ideal black body absorber. Note that this result is achieved for the object with a rather large electrical size $\beta_{0} R_{\mathrm{WH}}=30 \beta_{0} d=9.45$, which means that the circumference of the object is on the order of 10 wavelength. Fig. 6 


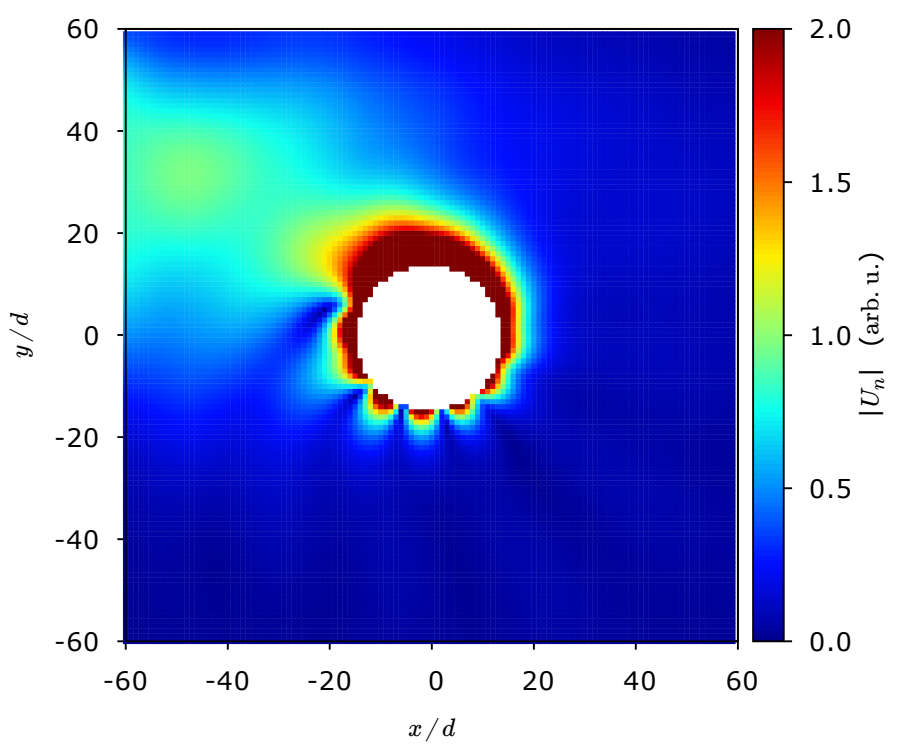

(a)

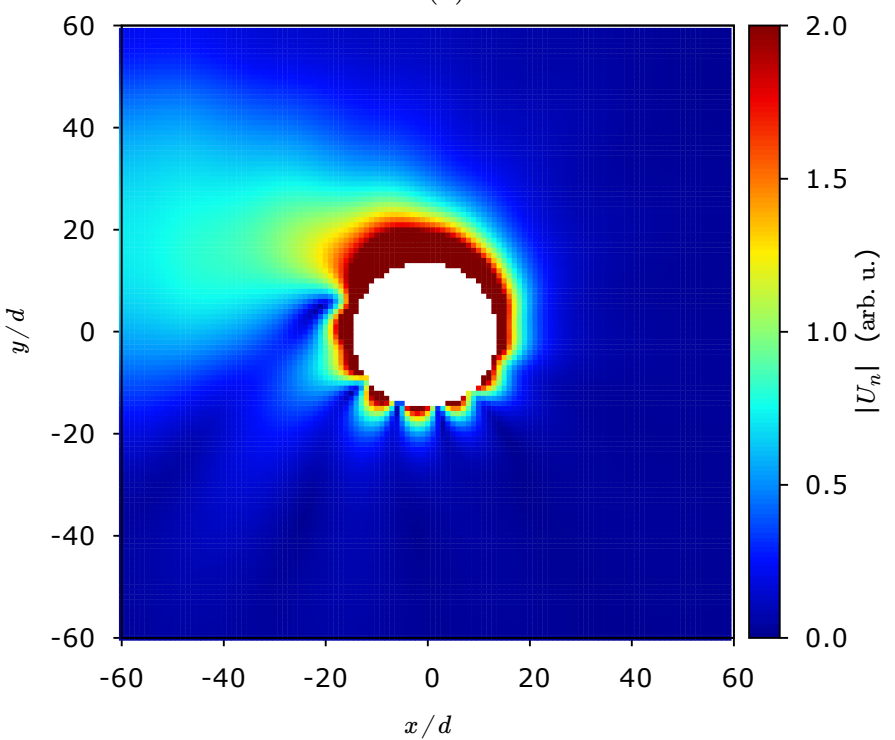

(b)

FIG. 7. (Color online) Trapping of a Gaussian beam by the metamaterial wormhole. Panel (a): The distribution of nodal voltage $\left|U_{n}\right|$ in the DPS plane. Panel (b): Same for the DNG plane. The radius of the wormhole is $R_{\mathrm{WH}}=15 d$. The Gaussian beam source (see Appendix B) is located in the DPS plane at $x=-60 d$, with its maximum at $y_{0}=30 d$ and the width $w=40 d$. The electrical parameters are $\beta_{0} d=0.1, \tan \delta=10^{-4}$.

also shows that there are practically no reflections from the front of the wormhole in the DPS region.

Fig. 7 illustrates trapping of nearby passing beams of radiation by the metamaterial wormhole. In this example, we have decreased the electrical cell size to $\beta_{0} d=0.1$ and the wormhole radius to $R_{\mathrm{WH}}=15 d$ in order to obtain a more pronounced effect. When illuminated by a plane wave (not shown in Fig. 7) the normalized absorption cross section of this object is $\sigma_{\text {norm }} \approx 2.7$, which means that the object's shadow radius is about $2.7 R_{\mathrm{WH}} \approx 40 d$.

Since the maximum of the incident Gaussian beam is at $y_{0}=30 d$, it falls within the "interception range" of the metamaterial superabsorber. We can see from Fig. 7 (a) that most of the energy of the beam is captured by the wormhole. After passing through the wormhole neck, the beam propagates in the DNG plane in the opposite direction [Fig. 7 (b)] and its amplitude decreases (and the width increases) due to diffraction, until the beam gets absorbed at the edge of the DNG domain.

Although the results of Fig. 6 and Fig. 7 confirm the presence of the superabsorption effect and illustrate the main phenomena associated with it, from the application point of view, it would be interesting to consider if the same effect could be demonstrated in a structure in which the number of the DNG cells was greatly reduced. Specifically, 


\begin{tabular}{|c|c|c|c|c|c|c|c|c|}
\hline$r / d$ & 30 & 29 & 28 & 27 & 26 & 25 & 24 & 23 \\
\hline$\beta_{0} d$ & 0.315 & 0.397 & 0.284 & 0.340 & 0.719 & 0.576 & 0.623 & 0.777 \\
\hline$Z_{0} / Z_{0}^{\text {DPS }}$ & 1 & 0.776 & 1.525 & 0.781 & 0.961 & 0.824 & 1.05 & 1.04 \\
\hline$\sigma_{\text {norm }}$ & - & 1.05 & 1.22 & 1.13 & 1.21 & 1.22 & 1.20 & 1.15 \\
\hline \hline$r / d$ & 22 & 21 & 20 & 19 & 18 & 17 & 16 & 15 \\
\hline$\beta_{0} d$ & 0.757 & 0.675 & 0.609 & 1.076 & 0.674 & 1.313 & 0.921 & 0.808 \\
\hline$Z_{0} / Z_{0}^{\text {DPS }}$ & 1.28 & 1.10 & 1.25 & 1.14 & 1.11 & 1.05 & 0.778 & 0.972 \\
\hline$\sigma_{\text {norm }}$ & 1.21 & 1.18 & 1.14 & 1.16 & 1.18 & 1.07 & 1.09 & 1.10 \\
\hline
\end{tabular}

TABLE I. Parameters of the concentric rings of the DNG cells forming the object: The relative inner ring radius $r / d$, the normalized propagation factor parameter $\beta_{0} d$, the normalized characteristic impedance $Z_{0} / Z_{0}^{\text {DPS }}$, and the normalized absorption cross section $\sigma_{\text {norm. }}$. The first column with $r=R_{\mathrm{obj}}=30 d$ lists the electrical parameters of the DPS plane.

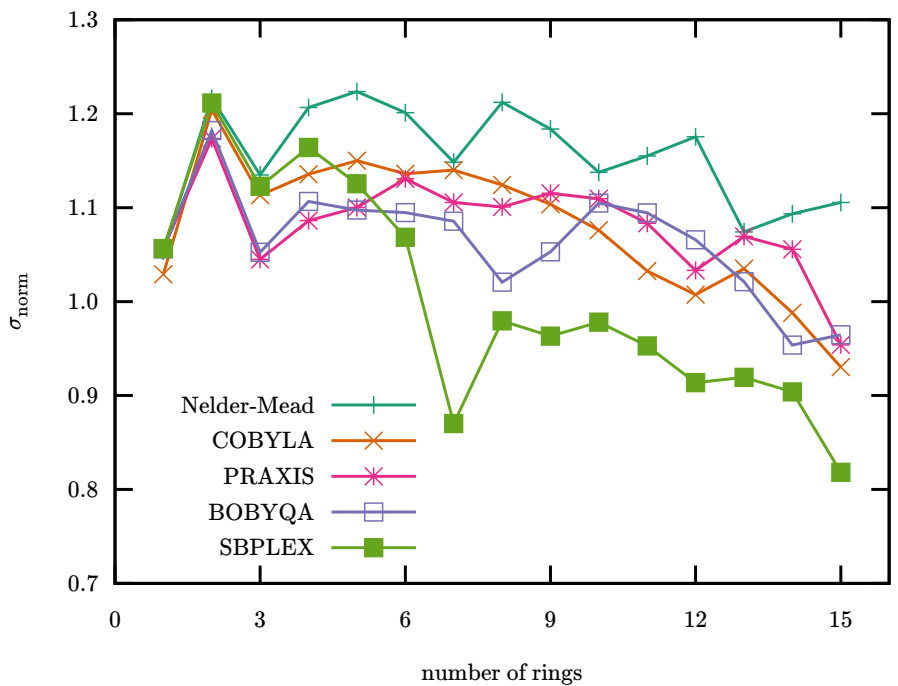

FIG. 8. (Color online) The normalized absorption cross section $\sigma_{\text {norm }}$ of the object formed by a number of concentric rings of the DNG cells with varying parameters as a function of the number of the object's rings. The radii of the rings are as in Tab. I. The electrical parameters of each ring are obtained by using a number of numerical optimization algorithms: Nelder-Mead, COBYLA, PRAXIS, BOBYQA, and SBPLEX. ${ }^{34}$

it is interesting to study the situation in which the DNG cells (in both DPS and DNG domains) occur only within a region of limited radius $r<R_{\mathrm{obj}}$, and with a wormhole of the radius $R_{\mathrm{WH}}<R_{\mathrm{obj}}$.

It is immediately understood that, in this case, the electrical parameters of the DNG cells in the DPS domain must vary with $r$. From the theoretical results for continuous media (Sec. II, the cylindrical case), one would expect that the normalized propagation factor $\beta_{0} d$ should vary with radius as $\beta_{0} d \propto \sqrt{\varepsilon_{z z} \mu_{\varphi}} \propto r^{-2}$ and the line impedance as $Z_{0} \propto \sqrt{\mu_{\varphi} / \varepsilon_{z z}} \propto r^{2}$. However, direct numerical FDTLM calculations show that, for cells with reasonably small (i.e., not too small) electrical thickness $\beta_{0} d \gtrsim 0.1$, the results obtained when using the profiles deduced from the continuous medium theory are far from being optimal. In fact, if $\beta_{0} d \approx 0.3$ (which is an attainable value from the practical point of view) in the uniform DPS region and at the object's border at $r=R_{\mathrm{obj}}$, then inside the object at, for example, $r=R_{\mathrm{obj}} / 2$, the same parameter must reach the value of $4 \times 0.3=1.2$, which is already too large for the cell to be considered electrically small, and thus the continuous medium approximation fails.

Therefore, in the following numerical simulations, an optimization approach for such a structure is used, the purpose of which is to establish some optimal variation profiles for $\beta_{0} d$ and $Z_{0}$ within the object. We consider an object with the outer diameter $R_{\mathrm{obj}}=30 d$, which is formed by up to 15 concentric rings of the DNG cells. The radii of these rings and the electrical parameters of the cells in these rings (as obtained by an optimization procedure) are listed in Tab. I. The table lists only the inner radii. The outer radius of a given ring is the inner radius of the previous ring, and so on, until the ring of the smallest radius is reached. The inner radius of the smallest ring is $r=R_{\mathrm{WH}}=15 d$, at which point the wormhole starts. In the DNG domain the region $R_{\mathrm{WH}}<r<R_{\mathrm{obj}}$ is filled with the cells whose electric parameters match to the parameters of the last ring.

The electrical parameters of the rings are optimized in order to maximize $\sigma_{\text {abs }}$ of the whole object. The optimization 


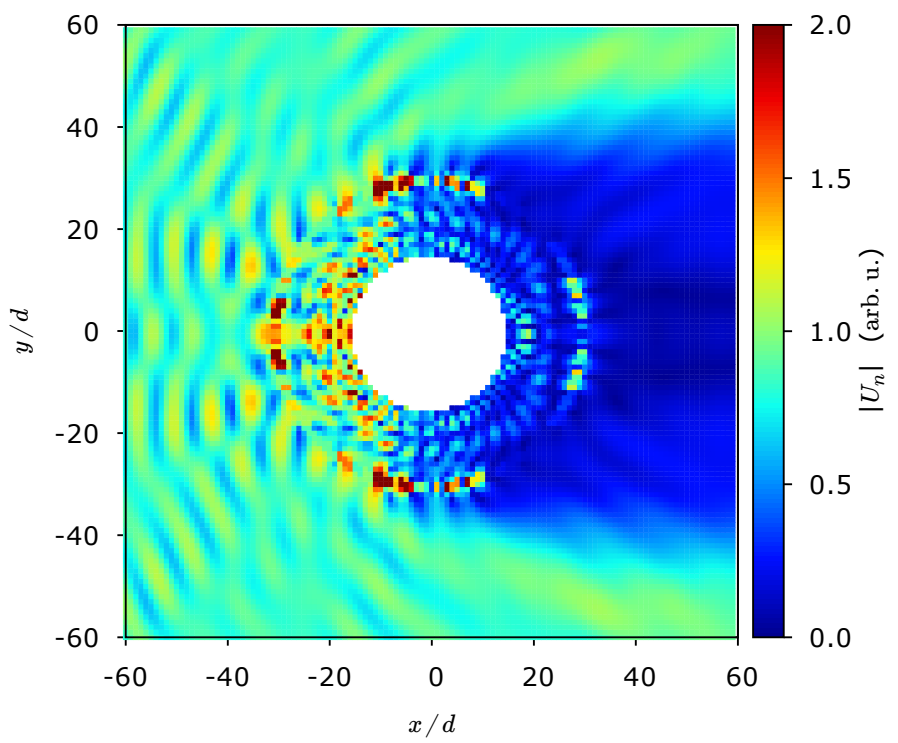

(a)

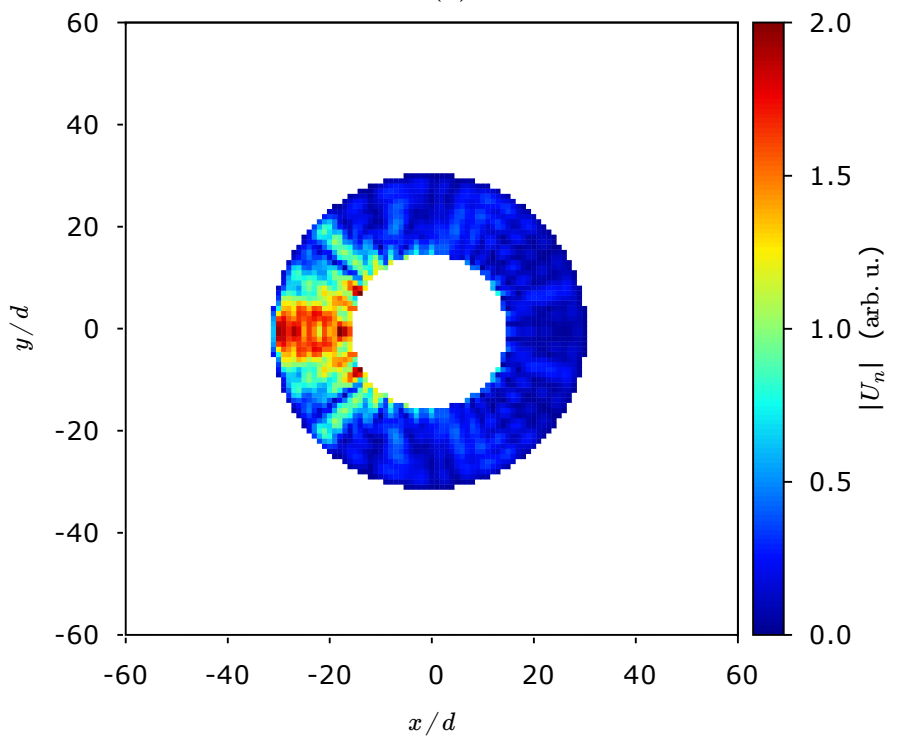

(b)

FIG. 9. (Color online) Distribution of the nodal voltage $\left|U_{n}\right|$ (defined in the same way as in Fig. 6) for an object composed by a set of concentric rings of the DNG cells of varying effective $\beta_{0}^{\text {DPS }} d$ and impedance $Z_{0}^{\text {DPS }}$ placed in the middle of the DPS domain [panel (a)] at $R_{\mathrm{WH}}<r<R_{\mathrm{obj}}$, whose inner ring is connected through the wormhole to the DNG domain [panel (b)] formed by a single uniform ring $R_{\mathrm{WH}}<r<R_{\mathrm{obj}}$ of the DNG cells. The structure is under the plane wave incidence of unitary amplitude. The object radius is $R_{\mathrm{obj}}=30 d$ and the wormhole radius is $R_{\mathrm{WH}}=15 d$. The electrical parameters in the DPS region where $r>R_{\mathrm{obj}}$ are $\beta_{0} d=0.315$, and $\tan \delta=10^{-4}$. The other parameters are listed in Tab. I.

is performed in gradual steps. First, a structure containing only a single ring with the largest radius is considered, with the wormhole starting at the inner edge of this ring. Because there is only one ring in the structure at this step, it is also the last ring, and the parameters of the cells in the DNG domain are matched to this ring. After the electrical parameters of this ring are optimized to maximize $\sigma_{\text {abs }}$, they are fixed, and the second ring is added to the structure (which becomes the new last ring) and the optimization of the electrical parameters of this ring is performed under the same goal. In this way, the parameters of all 15 rings are found. The optimization results are summarized in Fig. 8.

The FDTLM simulation results for two ring structures under plane wave incidence are shown in Figs. 9 and 10. As in Fig. 6, there are no cells in the white areas. As compared to the simple wormhole structure, the results of Fig. 9 show that this object has a much higher level of reflections, which means that some part of the incident power is lost due to these reflections, and, therefore, one may not expect to get a high effective $\sigma_{\text {abs }}$ for this object when a large 


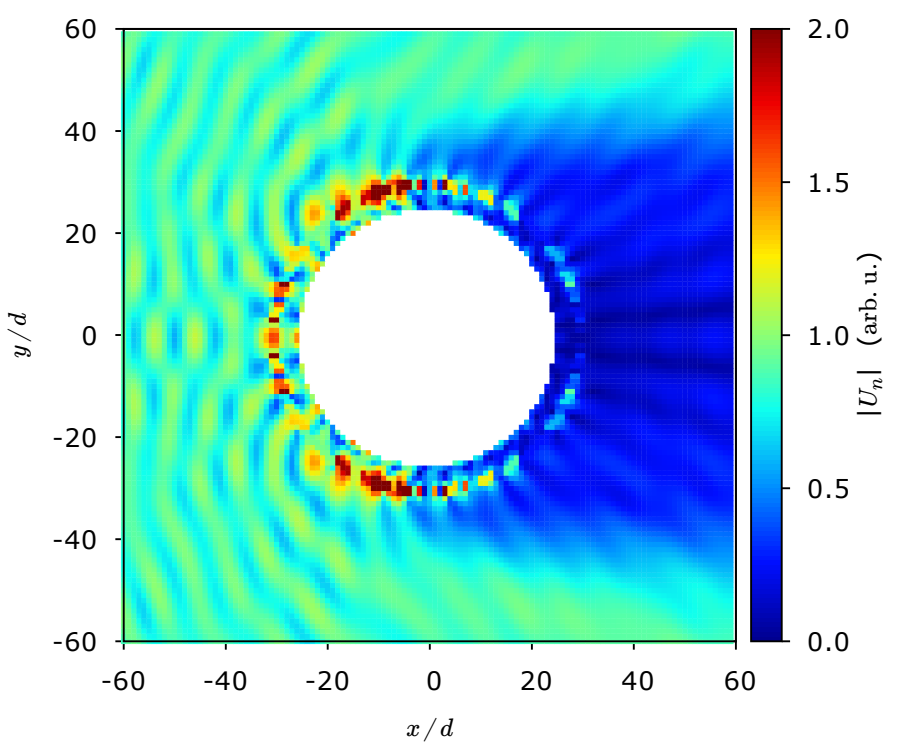

(a)

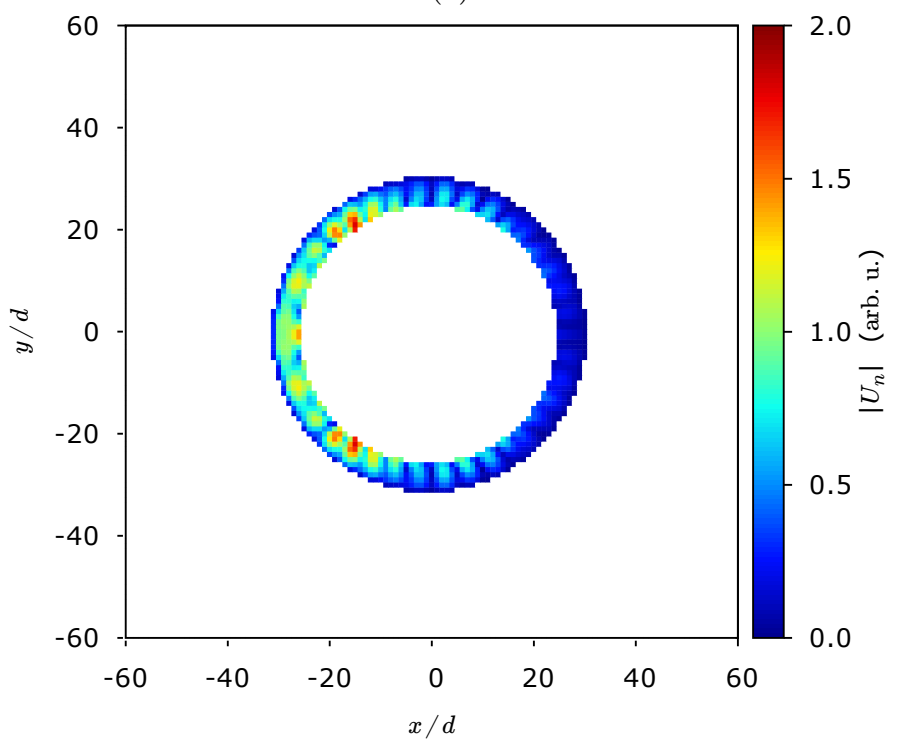

(b)

FIG. 10. (Color online) Distribution of the nodal voltage $\left|U_{n}\right|$ for an object composed by 5 concentric rings of the DNG cells in the DPS domain [panel (a)] and a single uniform ring $R_{\mathrm{WH}}<r<$ of the DNG cells in the DNG domain [panel (b)]. The structure is under the plane wave incidence of unitary amplitude. The ring radii and the electrical parameters of the DNG cells are listed in Tab. I.

number of rings is used.

In fact, further numerical simulations demonstrate (Fig. 8) that there is an optimal number of rings $n, 1<n<15$, at which $\sigma_{\text {abs }}$ attains a maximum. Fig. 8 shows the variation of the normalized absorption cross section, $\sigma_{\text {norm }}=$ $\sigma_{\mathrm{abs}} /\left(2 R_{\mathrm{obj}}\right)$, as a function of the object's rings number, as in the gradual optimization procedure described earlier. For the selected value of the $\beta_{0} d$ parameter in the DPS region: $\beta_{0} d=0.315$, the optimal ring number is $n=5$, with the rings' radii and the electrical parameters listed in the beginning of Tab. I (these parameters are obtained with the Nelder-Mead optimization algorithm from NLopt library ${ }^{34}$ ). The normalized absorption cross section in this case is $\sigma_{\text {norm }}=1.224$.

The distribution of the field around this object with a rather small number of the DNG cells is shown in Fig. 10. It is interesting that the performance of this object is still more than $20 \%$ higher than that of the black body absorber of the same diameter, and such an increase in the performance is achieved with just a small number of the DNG cells distributed around the perimeter of the wormhole, as compared with the case of Fig. 6, where a large number of the DNG cells distributed over the whole DNG domain is used. We expect that with an even better realization 
strategy that involves DNG cells of varying geometry and with better optimization procedures these results can be further improved. In fact, preliminary results obtained with a global optimization approach replacing the ring-by-ring approach (to be reported elsewhere) indicate that in the same setup one can achieve, at least, $\sigma_{\text {norm }} \approx 1.3$.

\section{CONCLUSIONS}

In this work, possible realizations of the superabsorbing metamaterial objects whose effective absorption cross section is significantly greater than the geometrical cross section have been studied theoretically and simulated numerically. The superabsorption effect has been modeled with the metamaterial TL-based structures that support effectively two-dimensional propagation of the electromagnetic waves. We have shown that in this model, a finite-size conjugateimpedance matched superabsorbing object can be equivalently represented with a wormhole structure formed by two electrically connected DNG and DPS domains. With respect to the waves propagating within the DPS domain, the wormhole appears as a conjugate-impedance matched absorber, while for the waves propagating in the DNG domain, the wormhole acts as a radiation source. The waves transmitted to the DNG domain through the wormhole neck are then absorbed at the edges of the DNG domain, where the open ports are terminated with matched loads.

By using the TL-based unit cells with the electrical size of $\beta_{0} d=0.1$ and the loss tangent value of tan $\delta=10^{-4}$, we have obtained the normalized absorption cross section about three times greater than that for the black body of the same size. We have demonstrated the trapping of nearby passing beams of radiation by the metamaterial superabsorbers that has been predicted earlier. ${ }^{20}$ For a larger wormhole structure with $\beta_{0} d=0.315$ and $\beta_{0} R_{\mathrm{WH}}=9.45$, the obtained normalized absorption cross section is $\sigma_{\text {norm }}=1.46$, which means that, even for objects with a relatively large circumference of about 10 wavelengths, the metamaterial wormhole superabsorber can outperform the black body absorber by about $50 \%$.

We have found that the superabsorption effect can be also observed in non-uniform structures with a smaller number of DNG cells, as compared to the complete wormhole structure. Namely, such an effect can be observed for a DNG metamaterial object that fits entirely within a region of a finite radius $r=R_{\text {obj }}$, such that $\beta_{0} R_{\text {obj }} \gg 1$. This is especially interesting for applications of the metamaterial superabsorbers as efficient harvesters of electromagnetic radiation which absorb more energy from an incoming plane wave than what is incident directly on their surface.

It is worth noting that the wormhole structure can be also used to demonstrate the narrow-band super-Planckian emitting property of the conjugate-impedance matched superabsorbers. ${ }^{18}$ In order to do this in practice, one will have to perform electric (Johnson-Nyquist) noise measurements in this structure under controlled thermal conditions. We reserve such a study for a future work.

\section{ACKNOWLEDGMENT}

The authors acknowledge support under the project Ref. UID/EEA/50008/2013, sub-project MMSUPER, financed by Fundação para a Ciência e a Tecnologia (FCT)/Ministério da Ciência, Tecnologia e Ensino Superior (MCTES), Portugal. S.I.M. acknowledges support from Fundação para a Ciência e a Tecnologia (FCT), Portugal, under Investigador FCT (2012) grant (Ref. IF/01740/2012/CP0166/CT0002).

\section{Appendix A: Scattering matrices of conjugate-impedance matched DPS and DNG cells}

The $S$-matrix of the square unit cell with the schematic depicted in Fig. 2 is a $4 \times 4$ matrix whose elements, $S_{m n}$, satisfy $S_{m n}=S_{n m}$ due to the reciprocity, and $S_{11}=S_{22}=S_{33}=S_{44}$ due to the symmetry of the unit cell. In addition, if the ports are numbered around the perimeter of the unit cell (for example, in the counterclockwise direction), the unit cell symmetry demands that $S_{12}=S_{14}, S_{13}=S_{24}$.

Note that because in the considered analytical model all four ports of the unit cell are equivalent to each other, the $S$-parameter matrix resulting from this model also must satisfy $S_{12}=S_{13}$, and thus all elements of the $S$-matrix can be expressed through just a pair of reflection and transmission coefficients $\mathcal{R}$ and $\mathcal{T}$ of an isolated unit cell, assuming that its four ports are connected to infinite transmission lines with characteristic impedance $Z_{0}$. The analytical expressions for $\mathcal{R}$ and $\mathcal{T}$ are

$$
\mathcal{R}=-\frac{4(\bar{Y}+2 \bar{Z}+2) \tan ^{2} \frac{\beta_{0} d}{2}-4 j \bar{Z}(\bar{Z}+\bar{Y}) \tan \frac{\beta_{0} d}{2}-\bar{Y} \bar{Z}^{2}-8 \bar{Z}+4 \bar{Y}+8}{\left(2 j \tan \frac{\beta_{0} d}{2}+\bar{Z}+2\right)\left(2 j(\bar{Y}+2(\bar{Z}+2)) \tan \frac{\beta_{0} d}{2}+\bar{Y} \bar{Z}+2 \bar{Y}+8\right)}
$$


and

$$
\mathcal{T}=\frac{8\left(\tan ^{2} \frac{\beta_{0} d}{2}+1\right)}{\left(2 j \tan \frac{\beta_{0} d}{2}+\bar{Z}+2\right)\left(2 j(\bar{Y}+2(\bar{Z}+2)) \tan \frac{\beta_{0} d}{2}+\bar{Y} \bar{Z}+2 \bar{Y}+8\right)},
$$

with $\bar{Z}=Z / Z_{0}$, and $\bar{Y}=Z_{0} Y$.

Respectively, the $S$-matrix elements of the unit cell are expressed as

$$
S_{m m}=\mathcal{R}, \quad S_{m n}=\mathcal{T}, \quad \forall m, n: m \neq n .
$$

When $\bar{Z}=\bar{Y}=0$, which is the case of the DPS unit cell, Eqs. (A1) and (A2) reduce to

$$
\mathcal{R}^{\mathrm{DPS}}=-\frac{1}{2} e^{-j \beta_{0} d}, \quad \mathcal{T}^{\mathrm{DPS}}=\frac{1}{2} e^{-j \beta_{0} d},
$$

which is the well-known result for the reflection and transmission coefficients at a junction of four equal TL segments. ${ }^{35}$

In addition, it is easy to verify that when the values of the loads $Z$ and $Y$ are taken in accordance with Eqs. (17) and (18) for the conjugate-impedance matched DNG network with negligible $\operatorname{loss}\left(\operatorname{Im} \beta_{0} \rightarrow 0\right)$ and the real-valued characteristic TL impedance $Z_{0}$, the reflection and transmission coefficients (A1) and (A2) reduce to

$$
\mathcal{R}^{\mathrm{DNG}}=-\frac{1}{2} e^{+j \beta_{0} d}, \quad \mathcal{T}^{\mathrm{DNG}}=\frac{1}{2} e^{+j \beta_{0} d},
$$

and thus the $S$-matrix of the conjugate-impedance matched DNG cell (with the same real TL impedance $Z_{0}$ ) satisfies

$$
S_{m n}^{\mathrm{DNG}}=\left(S_{m n}^{\mathrm{DPS}}\right)^{*} .
$$

This result can be also obtained directly from the relation between the impedance matrix $\mathbf{Z}$ (the $Z$-matrix) and the scattering matrix $\mathbf{S}$ of a single cell through the following expressions: $\mathbf{S}=\left(\mathbf{Z}+Z_{0} \mathbf{I}\right)^{-1} \cdot\left(\mathbf{Z}-Z_{0} \mathbf{I}\right)$ and $\mathbf{Z}=$ $Z_{0}(\mathbf{I}+\mathbf{S}) \cdot(\mathbf{I}-\mathbf{S})^{-1}$, where $\mathbf{I}$ is the unit $4 \times 4$ matrix, and the generalized conjugate-impedance match condition: ${ }^{19}$ $\mathbf{Z}^{\mathrm{DNG}}=\left(\mathbf{Z}^{\mathrm{DPS}}\right)^{\dagger}$ (here, the symbol ${ }^{\dagger}$ denotes the Hermitian conjugate).

\section{Appendix B: Implementation of FDTLM method}

Given the $S$-matrices of all DPS and DNG cells, the wave propagation in the whole structure can be studied by solving a system of linear equations for the unknown wave amplitudes in the ports of adjacent unit cells. Namely, if the DPS cells are numbered as $1 \ldots N$ and the DNG cells are numbered as $1 \ldots M$, the unknown complex amplitudes of the incident and reflected waves (which can be understood as the waves of electric voltage) in all ports of all cells can be collected into column vectors $\mathbf{V}^{\mathrm{inc}}=\left(a_{1}^{\mathrm{DPS}}, \ldots, a_{4 N}^{\mathrm{DPS}}, a_{1}^{\mathrm{DNG}}, \ldots, a_{4 M}^{\mathrm{DNG}}\right)^{T}$, and $\mathbf{V}^{\mathrm{ref}}=\left(b_{1}^{\mathrm{DPS}}, \ldots, b_{4 N}^{\mathrm{DPS}}, b_{1}^{\mathrm{DNG}}, \ldots, b_{4 M}^{\mathrm{DNG}}\right)^{T}$, respectively. The length of these vectors is $4(N+M)$ due to the fact that each cell has four ports, and, therefore, these vectors are composed of $N+M$ groups of four wave amplitudes belonging to each cell.

As is evident from the above definition, the vectors $\mathbf{V}^{\text {ref }}$ and $\mathbf{V}^{\text {inc }}$ satisfy

$$
\mathbf{V}^{\mathrm{ref}}=\mathbf{S} \cdot \mathbf{V}^{\mathrm{inc}}
$$

where $\mathbf{S}=\operatorname{diag}\left(\mathbf{S}_{1}^{\mathrm{DPS}}, \ldots \mathbf{S}_{N}^{\mathrm{DPS}}, \mathbf{S}_{1}^{\mathrm{DNG}}, \ldots \mathbf{S}_{M}^{\mathrm{DNG}}\right)$ is a block-diagonal matrix formed by the $4 \times 4$ scattering matrices of all the DPS and DNG cells in the wormhole structure.

On the other hand, because the adjacent ports in the neighboring cells are connected, the incident wave in one of such ports is essentially the reflected wave in the other and vice versa. Therefore,

$$
\mathbf{V}^{\text {inc }}=\mathbf{C} \cdot \mathbf{V}^{\text {ref }}
$$

where $\mathbf{C}$ is the so-called connection matrix. The elements of $\mathbf{C}$ are mostly zeros, with some elements $C_{m n}=1$, where $m$ and $n$ are such that the electric connection of the respective ports in a pair of adjacent cells demands that $V_{m}^{\text {inc }}=V_{n}^{\text {ref }}$. It is evident that $C_{m n}=C_{n m}$. The general structure of the connection matrix can be represented in the block matrix form as

$$
\mathbf{C}=\left(\begin{array}{ll}
{\left[\mathbf{C}_{\mathrm{DPS}}\right]_{(4 N \times 4 N)}} & {\left[\mathbf{C}_{\mathrm{WH}}\right]_{(4 N \times 4 M)}} \\
{\left[\mathbf{C}_{\mathrm{WH}}^{T}\right]_{(4 M \times 4 N)}} & {\left[\mathbf{C}_{\mathrm{DNG}}\right]_{(4 M \times 4 M)}}
\end{array}\right) .
$$


Note that besides connections between the DPS cells within the DPS domain represented by $\mathbf{C}_{\mathrm{DPS}}$, and similar connections between the DNG cells in the DNG domain represented by $\mathbf{C}_{\mathrm{DNG}}$, there are also connections between the DPS and DNG cells at the wormhole neck, which are taken into account by the off-diagonal blocks $\mathbf{C}_{\mathrm{WH}}$ and $\mathbf{C}_{\mathrm{WH}}^{T}$.

Additionally, the diagonal elements $C_{m m}$ with indices $m$ that correspond to unconnected ports at the edges of the structure can be set to a non-zero value in order to realize an absorbing boundary condition (ABC) which will imitate infinite continuation of the periodic DPS or DNG mesh. A good approximation for such ABC at the edge of the DPS or the DNG structure is $C_{m m}=\Gamma_{0}$, where

$$
\Gamma_{0}=\frac{Z_{\perp}-Z_{0}}{Z_{\perp}+Z_{0}},
$$

with $Z_{\perp}$ being the Bloch impedance for the wave in the DPS (or the DNG) mesh that impinges normally at the interface where the $\mathrm{ABC}$ is defined.

When external sources are present, Eq. (B2) must be modified in order to include the contribution to the incident waves due to such sources:

$$
\mathbf{V}^{\text {inc }}=\mathbf{C} \cdot \mathbf{V}^{\text {ref }}+\mathbf{V}^{\text {ext }}
$$

By combining Eq. (B1) with Eq. (B5) we find that

$$
(\mathbf{I}-\mathbf{C} \cdot \mathbf{S}) \cdot \mathbf{V}^{\text {inc }}=\mathbf{V}^{\text {ext }}
$$

In the simulations of the wormhole structure, Eq. (B6) is solved for $\mathbf{V}^{\text {inc }}$ for a given excitation vector $\mathbf{V}^{\text {ext }}$. Next, the vector $\mathbf{V}^{\text {ref }}$ is found from Eq. (B1). The vector of total voltages at the input ports of all unit cells is then obtained as $\mathbf{V}=\mathbf{V}^{\text {inc }}+\mathbf{V}^{\text {ref }}$, from which we can express the electric voltage at the middle point of $n$-th DPS unit cell, by using the equivalent network of Fig. 2 (with $Z=Y=0$ ):

$$
U_{n}^{\mathrm{DPS}}=\frac{\sum_{i=1}^{4} V_{4 n-4+i}}{4 \cos \frac{\beta_{0} d}{2}}, \quad 1 \leq n \leq N,
$$

and, at $m$-th DNG cell, as

$$
U_{m}^{\mathrm{DNG}}=\frac{\sum_{i=1}^{4} V_{N+4 m-4+i}}{\left(4+\frac{Y Z}{2}\right) \cos \frac{\beta_{0} d}{2}+j\left(Y Z_{0}+\frac{2 Z}{Z_{0}}\right) \sin \frac{\beta_{0} d}{2}}, \quad 1 \leq m \leq M,
$$

which reduces to

$$
U_{m}^{\mathrm{DNG}}=\frac{\sum_{i=1}^{4} V_{N+4 m-4+i}}{4 \cos \frac{\beta_{0} d}{2}}, \quad 1 \leq m \leq M,
$$

when the load impedance $Z$ and the load admittance $Y$ are given by Eqs. (17) and (18).

Eqs. (B1)-(B9) constitute the main theoretical formulation of the employed FDTLM method. Note that the external source vector $\mathbf{V}^{\text {ext }}$ can be initialized in many different ways, allowing for a number of excitation scenarios to be studied. For instance, the excitation by an incident 2D Gaussian beam can be modeled by setting up the elements $V_{n}^{\text {ext }}$ that correspond to the open ports at one of the four edges of the DPS domain (for instance, at $x=-N_{x} d / 2$, where $N_{x}$ is the number of cells across the whole structure along the $x$-axis, with $x=y=0$ being at the middle of the DPS domain) to values proportional to $e^{-j k_{\mathrm{t}} y-\left(y-y_{0}\right)^{2} / w^{2}}$, with $y_{0}, w$, and $k_{\mathrm{t}}$ being the parameters of the beam, and $y$ being the coordinate along the edge. Such a source will produce a Gaussian beam that propagates in the DPS domain in the direction defined by the wave vector $\mathbf{k}=\left(k_{x}, k_{y}\right)$, where $k_{y}=k_{\mathrm{t}}$ and $k_{x}$ is given by Eq. (10). The parameter $w$ defines the width of the beam, and the parameter $y_{0}$ sets the initial location of its maximum.

The external source can also be defined for an effective plane wave excitation scenario. Moreover, in this case it is possible to set up the source in a way that mimics electromagnetic excitation of a body by equivalent Huygens 
sources - pairs of orthogonal electric and magnetic dipole moments defined on a surface fully enclosing the object under study. ${ }^{36}$ Because the field of such sources vanishes outside the enclosed domain, the outside field is just the field scattered by the body, i.e., excitation of an object by such sources allows for a straightforward determination of the field scattered by the object.

In an FDTLM simulation, such a Huygens source can be constructed in the following way. First, based on the results of the analytical model of Sec. III B, a vector $\mathbf{V}^{\text {inc }}=\mathbf{V}_{B}^{\text {DPS }}$ that corresponds to a Bloch wave solution in a uniform DPS domain with no scatterers (i.e., without the wormhole or any other irregularities) is formed. Second, a mask $\mathbf{M}=\operatorname{diag}\left(M_{n}\right), M_{n} \in\{0,1\}$, is applied by calculating the product $\mathbf{M} \cdot \mathbf{V}_{\mathrm{B}}^{\text {DPS }}$ such that it filters out the elements that relate to the unit cells outside the domain that we wish to be enclosed by the Huygens source. Finally, the Huygens source vector $\mathbf{V}_{\mathrm{H}}^{\text {ext }}$ that creates the plane-wave-like incident field inside the enclosed domain and the zero field outside is found as

$$
\mathbf{V}_{\mathrm{H}}^{\mathrm{ext}}=\left(\mathbf{I}-\mathbf{C}_{0}^{\mathrm{DPS}} \cdot \mathbf{S}_{0}^{\mathrm{DPS}}\right) \cdot \mathbf{M} \cdot \mathbf{V}_{\mathrm{B}}^{\mathrm{DPS}}
$$

where $\mathbf{C}_{0}^{\text {DPS }}$ and $\mathbf{S}_{0}^{\text {DPS }}$ are the connection and scattering matrices of the uniform, unperturbed DPS domain. In order to determine the wormhole behavior under such excitation, the source (B10) is substituted into Eq. (B6) and the resulting matrix system is solved for $\mathbf{V}^{\text {inc }}$. The obtained solution will relate to the total (incident plus scattered) field in the DPS region enclosed by the Huygens source and to the transmitted field in the DNG region, while in the DPS region outside the enclosed domain, it will relate only to the scattered field.

Finally, the total electric power that enters into the wormhole under a given excitation and becomes eventually absorbed due to the ABCs at the edges of the DNG domain can be expressed as (we understand the time-harmonic voltages as rms values)

$$
P_{\mathrm{abs}}=\operatorname{Re}\left[\frac{1}{Z_{0}} \mathbf{V}^{\mathrm{inc}} \cdot\left(\begin{array}{cc}
\mathbf{0} & -\mathbf{C}_{\mathrm{WH}} \\
\mathbf{C}_{\mathrm{WH}}^{T} & \mathbf{0}
\end{array}\right) \cdot \mathbf{V}^{\mathrm{ref}}\right] \text {, }
$$

and the effective absorption cross section $\sigma_{\text {abs }}$ of the wormhole is calculated as

$$
\sigma_{\mathrm{abs}}=\frac{P_{\mathrm{abs}}}{\Pi_{\mathrm{inc}}},
$$

where $\Pi_{\text {inc }}$ is the density of the incident power flux, which is determined by the amplitude of the incident wave. For example, for an incident Bloch wave propagating in the DPS domain along the $x$-axis and characterized by the incident wave amplitude $V_{0}^{\text {inc }}$ in the input ports at the edge $x=-N_{x} d / 2$, where the wave enters the structure,

$$
\Pi_{\text {inc }}=\frac{1}{Z_{0} d}\left(1-\left|\Gamma_{0}\right|^{2}\right)\left|V_{0}^{\text {inc }}\right|^{2} .
$$

Note that in the 2D scattering problem that we consider, $\sigma_{\text {abs }}$ has the dimension of a length (and the physical meaning of a characteristic diameter) rather than an area (as in the $3 \mathrm{D}$ case).

* stas@co.it.pt

${ }^{1}$ C. F. Bohren and D. R. Huffman, Absorption and Scattering of Light by Small Particles (Weinheim: Wiley-VCH Verlag GmbH \& Co. KGaA, 2007).

2 S. Tretyakov, Plasmonics 9, 935-944 (2014).

3 M. I. Tribelsky and B. S. Luk'yanchuk, Phys. Rev. Lett. 97, 263902 (2006).

4 Z. Ruan and S. Fan, Phys. Rev. Lett. 105, 013901 (2010).

5 M. I. Tribelsky, EPL 94, 14004 (2011).

6 Z. Ruan and S. Fan, Appl. Phys. Lett. 98, 043101 (2011).

7 X. Fan, W. Zheng, and D. J. Singh, Light: Sci. Appl. 3, e179 (2014).

8 N. Mohammadi Estakhri and A. Alù, Phys. Rev. B 89, 121416(R) (2014).

9 A. E. Miroshnichenko and M. I. Tribelsky, Phys. Rev. Lett. 120, 033902 (2018).

10 A. Schelkunoff and H. T. Friis, Antennas: Theory and Practice (New York: Wiley, 1952), p. 27.

11 S. A. Tretyakov, S. Maslovski, and P. A. Belov, IEEE Trans. on Antennas and Propagation 51(10), 2652 (2003).

12 A. Alu and S. Maslovski, IEEE Trans. on Antennas and Propagation 58(5), 1436 (2010).

13 L. J. Chu, J. Appl. Phys. 19, 1163 (1948).

14 R. F. Harrington, IRE Trans. on Antennas and Propagation AP-6, 219 (1958).

15 J. Ng, H. Chen, and C. T. Chan, Opt. Lett. 34, 644 (2009). 
16 C. A. Valagiannopoulos, J. Vehmas, C. R. Simovski, S. A. Tretyakov, and S. I. Maslovski, Phys. Rev. B 92, 245402 (2015).

17 V. G. Veselago, Sov. Phys. Uspekhi. 10, 509 (1968).

18 S. Maslovski, C. Simovski, and S. Tretyakov, New J. Phys. 18, 013034 (2016).

19 S. I. Maslovski, C. R. Simovski, and S. A. Tretyakov, in Proceedings of SPIE, Metamaterials X, edited by A. D. Boardman et al. (SPIE, ISBN: 9781510601284, 2016), vol. 9883, p. 98830 O.

20 S. Maslovski, in Proceedings of 2016 10th International Congress on Advanced Electromagnetic Materials in Microwaves and Optics (METAMATERIALS) (IEEE Xplore, ISBN: 978-1-5090-1803-1, 2016), p. 220.

21 E. E. Narimanov and A. V. Kildishev, Appl. Phys. Lett. 95, 041106 (2009).

22 Q. Cheng, T. J. Cui, W. X. Jiang, and B. G. Cai, New J. Phys. 12, 063006 (2010).

${ }^{23}$ H. Chen, R.-X. Miao, and M. Li, Opt. Express 18, 15183 (2010).

24 Y. Yang, L. Y. Leng, N. Wang, Y. Ma, and C. K. Ong, J. Opt. Soc. Am. A 29, 473 (2012).

25 J. B. Pendry, D. Schurig, and D. R. Smith, Science 312(5781), 1780 (2006).

${ }^{26}$ W. J. Padilla, D. N. Basov, and D. R. Smith Materials Today 9(78), 28 (2006).

27 G. V. Eleftheriades, Materials Today 12(3), 30 (2009).

28 A. Grbic and G. V. Eleftheriades, J. Appl. Phys. 98, 043106 (2005).

29 P. Alitalo, S. Maslovski, and S. Tretyakov, J. Appl. Phys. 99, 064912 (2006).

30 P. Alitalo, S. Maslovski, and S. Tretyakov, J. Appl. Phys. 99, 124910 (2006).

31 CST MICROWAVE STUDIO (R) - 3D EM Simulation Software. Cst.com. http://www.cst.com/products/cstmws [June 2018].

32 ANSYS HFSS: High Frequency Electromagnetic Field Simulation. Ansys.com. https://www.ansys.com/products/electronics/ansys-hfss [June 2018].

${ }^{33}$ H. Jin and R. Vahldieck, IEEE Trans. on Microwave Theory and Techniques 20(12), 2207 (1992).

34 S. G. Johnson. The NLopt nonlinear-optimization package. Ab-initio.mit.edu. http://ab-initio.mit.edu/nlopt [June 2018].

35 C. Christopoulos, The Transmission-Line Modeling (TLM) Method in Electromagnetics, (Morgan \& Claypool Publishers, Synthesis Lectures on Computational Electromagnetics \#7, 2006), p. 32.

${ }^{36}$ I. V. Lindell, S. A. Tretyakov, and K. I. Nikoskinen, Electromagnetics 20(3), 233 (2000). 